\title{
PRINCIPAIS ESPÉCIES DE FORAMINÍFEROS E BRIOZOÁRIOS DO ATOL DAS ROCAS
}

\author{
ALTAIR DE JESUS MACHADO* \& FACELUCIA BARROS CORTES SOUZA*
}

\begin{abstract}
FORAMINIFERA AND BRYOZOA FROM ATOL DAS ROCAS, BRAZIL. In the biological reserve of the Atol das Rocas, a varied microfauna of foraminifera occur. From the 74 identified species, five are frequent in all analysed samples, and are: Archaias angulatus, Amphistegina lessonii, Gypsina vesicularis, Sorites marginalis and Heterostegina suborbicularis. The genus Triloculina has a greater diversity in the samples from the Farol island and from the reef top. the reef more exposed areas during low tide. The Miliolids and the Rotaliids are predominant, reflecting typical normal marine to hypersaline waters. The microfauna association showed different characteristics in various reef sub-environments. From the 35 analysed samples only 27 have bryozoan fragments. The number of the species (23) is less than that of foraminifera (74), but still indicates that the Atol das Rocas reef environment is favorable for the development of these organisms. The average frequency of 9 fragments per $10 O g$ of sediment is also very low, and this can be due to the wave energy impact, in shallow waters, causing the breakage of the animal colonies. From the 23 species, the following genus predominate: Scrupocellaria, Schizoporella, Smittina and Celleporaria, being the species Onychocella americana and Reptadeonellas violacea the most common, with of 5\%.
\end{abstract}

Keywords: Foraminifera, bryozoans, Atol das Rocas

\begin{abstract}
RESUMO Na reserva biológica do Atol das Rocas ocorre uma abundante e bem diversificada microfauna de foraminíferos. Do total de 74 espécies identificadas, 5 s3o freqüentes, e são elas: Archaias angulatus, Amphistegina lessonii, Gypsina vesicularis, Sorites marginalis e Heterostegina suborbicularis. O gênero Triloculina apresenta maior diversidade nas amostras da ilha do Farol e do topo recifal, zonas que são mais expostas durante a baixa-mar. Os Miliolídeos e os Rotaliideos são predominantes, refletindo o ambiente típico de águas marinhas normais a hipersalinas. A composição da microfauna de foraminíferos apresenta diferentes características nos diversos sub-ambientes recitais. Das 35 amostras, apenas 27 contem fragmentos de briozoários. O número de espécies (23), muito embora proporcionalmente inferior a dos foraminíferos (74), sugere que o ambiente do Atol das Rocas é favorável ao desenvolvimento desses animais. A sua freqüência média de 9 fragmentos/1 OOg de sedimento é relativamente baixa, e isto pode ser atribuído ao impacto das ondas, em águas rasas, provocando a fragmentação das colônias. Das 23 espécies, predominam os queilostomos dos gêneros Scrupocellaria, Schizoporella, Smittina e Celleporaria sendo que as espécies Onychocella americana e Reptadeonella violacea, são as mais comuns, ocorrendo com freqüência de $5 \%$.
\end{abstract}

Palavras Chave: Foraminíferos, briozoários, Atol das Rocas

INTRODUÇÃO O anel recifal do Atol das Rocas é descrito como uma estrutura constituída de espessas crostas *de algas coralinas e uma contribuição menor do coral Siderastrea stellata. Outros organismos, como os moluscos, foraminíferos incrustantes e briozoários também estão presentes (Kikuchi \& Leão, 1990; Kikuchi \& Abdon, 1991). Até então o conhecimento das características específicas dos foraminíferos limitava-se, apenas, à classificação de espécimens isolados de 4 amostras de sedimento recente, realizada por Tinoco (1965/6 e 1972), que constatou a dominância das espécies Amphistegina radiata, Archaias angulatus, Gypsina vesicularis e Peneroplis sp. As formas aglutinantes e os Miliolídeos foram considerados raros e os planctônicos estavam representados apenas pela espécie Globigerinoides trilobus.

No estudo dos organismos incrustantes do Atol das Rocas, Machado et al. $(1991,1992)$ encontraram apenas os briozoários incrustantes Antropora typica, Hippothoa flagelum, Schizoporella sp. e Tremogasterina sp. A participação dos briozoários nos sedimentos do Atol era, até o presente, desconhecida.

Este trabalho visa, portanto, mostrar em maiores detalhes a composição da microfauna de foraminíferos e briozoários nas areias carbonáticas do Atol das Rocas, incluindo a distribuição qüântico-qualitativa das 74 espécies de foraminíferos e 21 espécies de briozoários isoladas em 37 amostras de sedimento superficial. As espécies identificadas caracterizam o ambiente como marinho litorâneo normal a hipersalino, com águas quentes, bem agitadas, sob a influência da Corrente do Brasil.

ÁREA DE ESTUdO E METODOLOGIA O Atol das Rocas está situado a 144 milhas náuticas à leste da costa nordestina, entre as coordenadas de $3^{\circ} 51^{\prime}$ de latitude $\mathrm{S}$ e $33^{\circ} 49^{\prime}$ de longitude $\mathrm{W}$, e a 80 milhas a oeste do Arquipélago de Fernando de Noronha (Figura 1). Estudos recentes (Kikuchi \& Leão, 1990; Kikuchi \& Abdon, 1991) descrevem o Atol como um anel recifal, instalado sobre um monte submarino do tipo "guyot". Este anel é constituído, do ponto de vista morfológico, de terraços escalonados que represam as águas durante as marés baixas (Figura 1). Essas águas formam um sistema de piscinas (com $5 \mathrm{~m}$ de profundidade máxima), deixando visíveis 2 ilhas arenosas, sendo a maior delas a ilha do Farol. A porção norte do anel está sempre submersa, dando origem à laguna recifal. As águas oceânicas têm acesso ao interior do recife através do canal Barretinha e do Salão (Figura 2).

De acordo com informações do INPE - NATAL (Kikuchi \& Abdon, 1991), a salinidade das águas, medida na área, apresenta valores de $36 \%$; o $\mathrm{pH}$ de 8,5 e a tempera- 


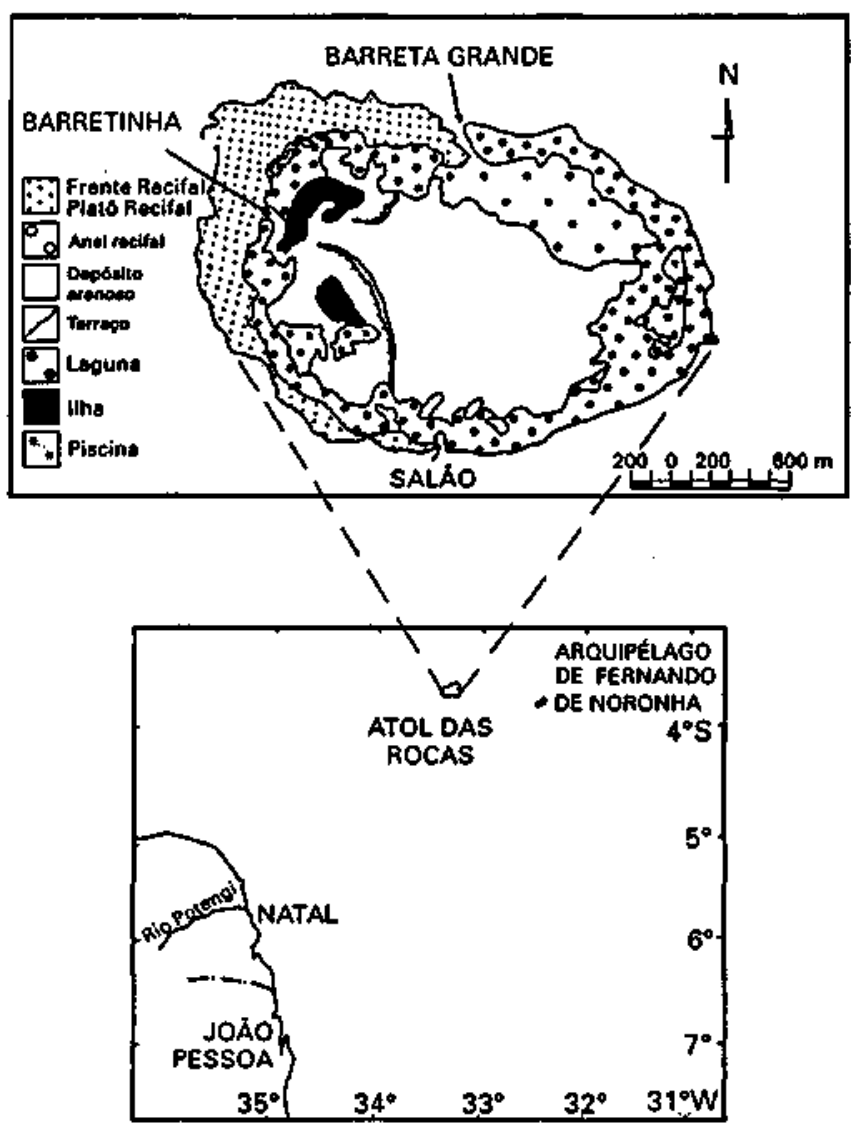

Figura 1 - Localização da área de estudo - Atol das Rocas (adaptado de Kikuchi \& Abdon, 1991)

Figure 1 - Location of study area - Atol das Rocas (modified from Kikuchi and Abdon, 1991) tura variável em torno de $27,5^{\circ} \mathrm{C}$, durante a maré de enchente.

Segundo dados de Kikuchi \& Leão (1991) toda a área recifal está recoberta por sedimentos do tipo areia cascalhosa carbonática algal, sendo, entretanto, um pouco mais fina na parte SO do depósito arenoso (amostras 36 e 35).

Das 37 amostras de sedimento coletadas nos terraços, piscinas e ilha do Farol (Figura 2), foram isolados os 300 primeiros foraminíferos e a totalidade de fragmentos de colônias de briozoários. No momento da coleta foi adicionado formol a $5 \%$ com água do mar, para fixação do protoplasma dos foraminíferos vivos.

No laboratório, após a lavagem em água corrente, adicionou-se às amostras o corante Rosa de Bengala para facilitar a identificação e contagem das formas vivas.

FORAMIIMÍFEROS DO ATOL DAS ROCAS A perfeita adaptação desses organismos ao ambiente recifal do Atol das Rocas se observa através do elevado índice de diversidade que varia de a 2,1 a 10,5, conforme ilustra o diagrama de Murray (1973) (Figura 3).

A distribuição das espécies em função da sua localização (tabelas 1 e 4), coloca em evidência que a maior diversidade ocorre nas piscinas do topo recifal, com o máximo de 45 espécies.

Esta adaptação se reflete também na predominância de testas de cor branca leitosa (calcária porcelânica), que são típicas de fundos calcários de águas quentes e rasas na província das índias Ocidentais (Boltovskoy 1965). As formas aglutinantes são raras (Textularia agglutinans, Textularia gramem e Textularia candeiana).

Relação mortos/vivos A observação do protoplasma corado, seja através da abertura, ou pela transparência da testa, permitiu considerar como componentes da biocenose, no momento da coleta, as espécies Ammonia beccarii, Amphistegina lessonii, Archaias angulatus, Borelispulchra,

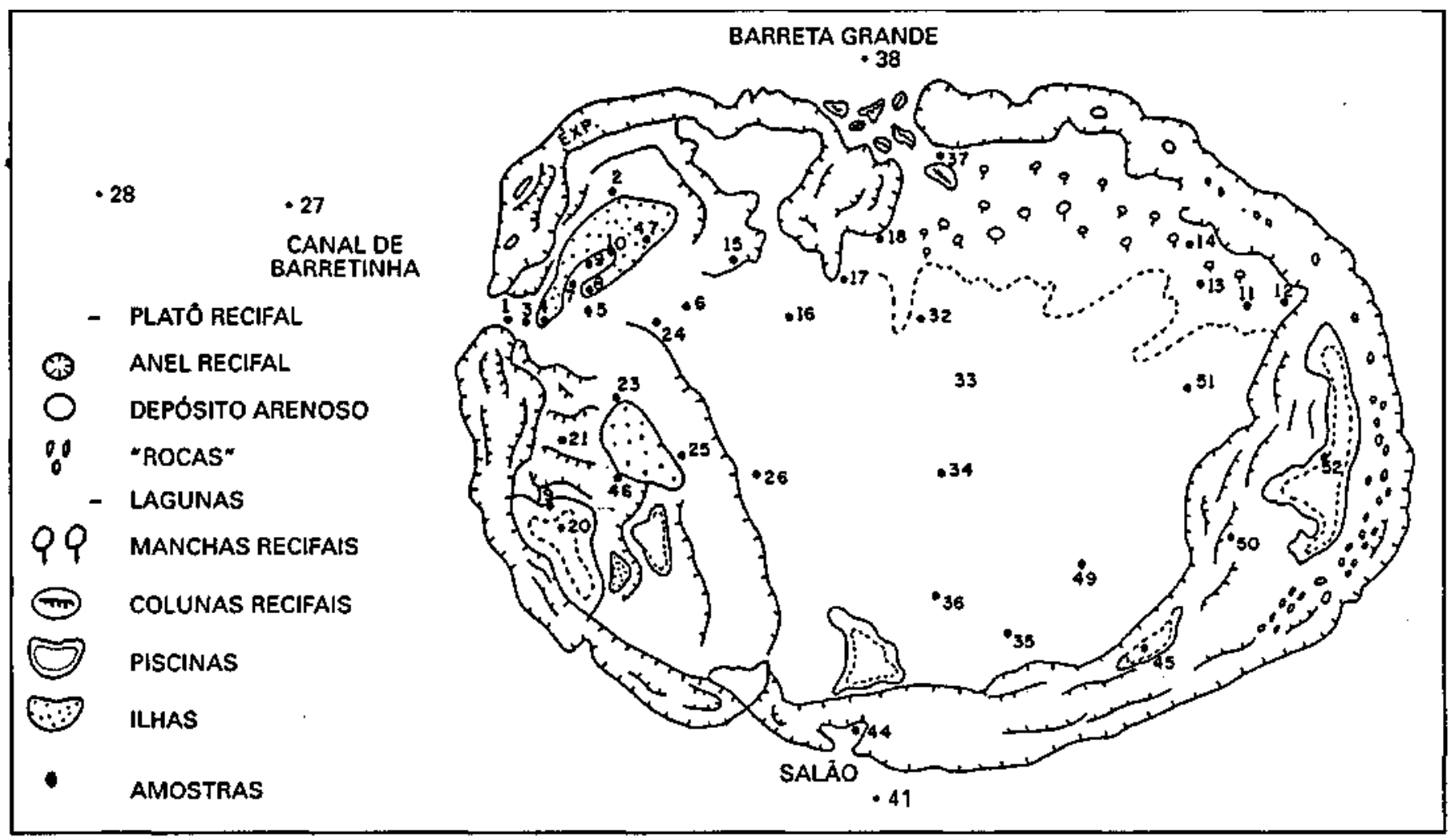

Figura 2 - Localização das amostras - Atol das Rocas (Abril 199I)

Figure 2 - Location of the samples - Atol das Rocas (April 1991) 


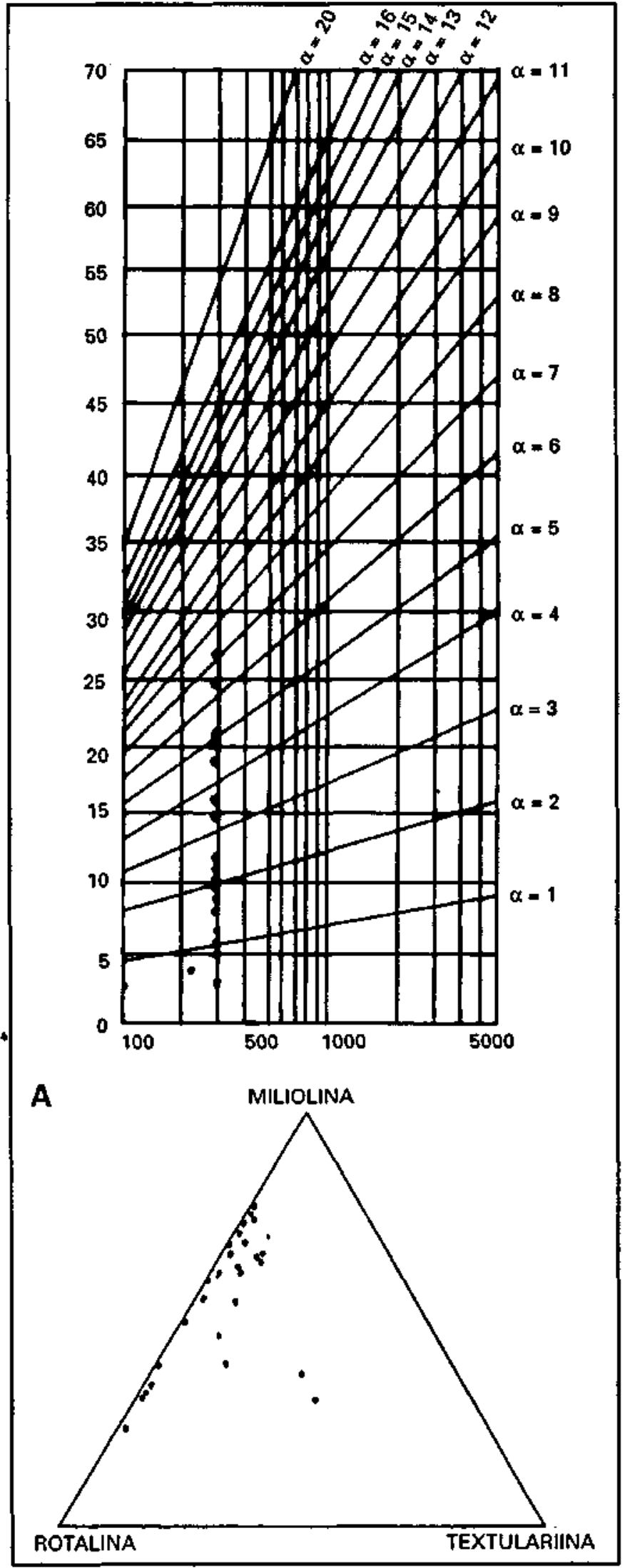

Figura 3 - índice de diversidade de acordo com Murray (1973). a. Diagrama triangular das relações entre as subordens de Foraminiferos (Murray, 1973)

Figure 3 - Diversity Index based on Murray (1973). a. Triangular diagram of the relations between sub-orders of Foraminifera besed on Murray (1973)
Gypsina vesicularis, Heterostegina suborbicularis, Sorites marginalis, Triloculina reliculata $f$. carinata, Triloculina reticulata $f$. bicarinata, Triloculina lutea e Triloculina subrotunda, entre os bentônicos e, Globigerinoides ruber entre os planctônicos (Tabela 2). As espécies citadas acima, exceto a Triloculina subrotunda e a Globigerinoides ruber, encontram-se misturadas com espécimens mal preservados. As espécies Quinqueloculina microcostulata, Quinqueloculina horrida e Dentosmina enoplostoma também apresentam mistura semelhante. Este fato indica a ação do retrabaIhamento e fragmentação dos espécimens.

Alguns exemplares de Archaias, Sorites e Triloculina (espécie reticulata $f$. bicarinata) apresentam-se retorcidos. Essa irregularidade pode ser de origem ecológica causada pela influência do ambiente. Segundo Boltovskoy (1965), as anormalidades nas carapaças são produzidas freqüentemente quando uma mudança rápida nas condições de vida (salinidade, alimentação, temperatura, conteúdo de alguns elementos químicos, atividade humana, etc.), conduz a uma variação no desenvolvimento anormal das carapaças. De um modo geral é muito difícil ou geralmente impossível dizer com precisão quais são as causas ecológicas que têm provocado as anormalidades nas carapaças. Podemos supor que os casos mais freqüentes são a mudança de salinidade e provavelmente as condições alimentares. Muitas vezes, devido a essa anormalidade encontrada nas carapaças dos foraminíferos, essas formas que apresentam irregularidade são descritas como "espécies novas".

Varias espécies apresentam formas jovens misturadas com as formas adultas, mais antigas, evidenciando contemporaneidade do material.

Relação planctônicos/bentônicos $\mathrm{O}$ índice de formas planctônicas é muito baixo, variando entre $01 / 300$ e 12/300. A distribuição das formas planctônicas (Figura 4) mostra que a freqüência máxima de $4 \%$ ocorre na parte sul do depósito arenoso. As principais espécies planctônicas encontradas foram Globigerinoides trilobus (dominante), Globigerinoides ruber, Globigerinoides aequilateralis, Globorotalia menardii e Globigerina bulloides.

A espécie Globigerinoides trilobus é assinalada como típica da Corrente do Brasil e das águas tropicais (Boltovskoy, 1965).

Principais espécies de foraminíferos e sua distribuição Archaias angulatus foi considerada a espécie dominante no Atol das Rocas pela sua freqüência elevada $(69 \%)$ e pela sua presença em todas as amostras (Tabela 2). Além de Archaias angulatus apenas Amphisiegina lessonii, Sorites marginalis e Triloculina subrotunda atingem freqüência superior a 2\%. De acordo com Murray (1973), as principais espécies citadas são características de suporte sedimentar (fundos calcários), suporte algal e recifes de coral. Archaias angulatus é adaptada às águas rasas e agitadas (Figura 5 e 8). Amphistegina lessonii requer águas com salinidade superior a $34 \%$, temperatura entre $25^{\circ}$ e $26^{\circ} \mathrm{C}$ e profundidade maior do que a espécie precedente. Isto explica as freqüências mais baixas de A lessonii (Figura 6 e 8). A espécie Sorites marginalis confirma no Atol a sua preferência ao habitat lagunar, pouco profundo $(0-35 \mathrm{~m})$ e o suporte algal. Ela vive em águas com salinidade superior a $37 \% \mathrm{e}$ temperatura entre $18^{\circ}$ e $26^{\circ} \mathrm{C}$ (Figura 7 e 8 ).

A microfauna leste e oeste A distribuição das espécies evidencia uma diferença na microfauna entre as partes leste e oeste do topo do recife, que se verifica através do número e da composição específica entre as duas zonas. A diversidade é maior à oeste do que à leste da área estudada, de acordo com a Tabela 4. No depósito arenoso (Tabela 5), as espécies Peneroplis bradyi, Peneroplis discoideus, 


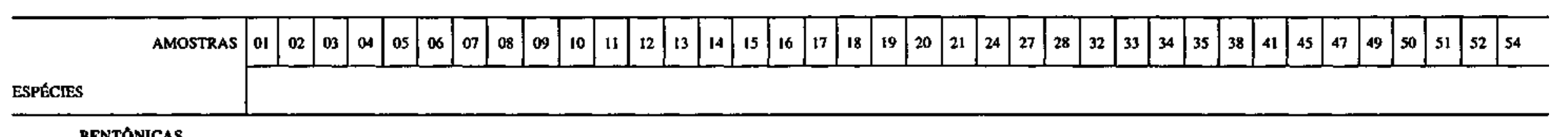

\section{BENTÓNICAS \\ Archaias angulasus}

Ampahistegina lessonit

Sorises marginalis

Trilaculino subrowurda

Heterostegina suborbicularis

Triloculina trigonuta

Borefis pulchro

Gypsina vesicularis

Tritoculina reticulata f, earimated

Trilocultina reticulata f, topica

Peneroplit carinatus

Triloculina emadrilateratis

Triloculina reticulaka f, bicarinata

Trilloculina ablonga

Discorbis mira

Peneroplis pertusus

Quinqueloculina of intplexa

Spiroloculina antitartem

Petseroplis proteus

Trilocultina sommer

Poroppontides lateralis

Pyrgo subsphtaerica

Pyrgo elongara

Peneroplis discoidens

Tresomphatus bulloides

Dentostamina enoplostama

Peneroplis bradyi

Triboculina latea

Tribowling linaedre

Elphidium sagrum

Sipharina pulchra

Textularia agglutinats

Triloculita platcians

Quinqueloculina seminulum

Qringuelaculina angulasa

Spinolorulina aspersia

Triloculititu elongasa

Articulina multilocularis

Elphidium galvestonensis

Miliolinella suborbicularis

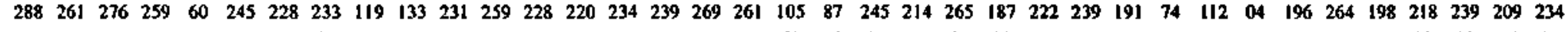
$\begin{array}{llllllllllllllllllllllllllllllllllllllllllllll}08 & 22 & 14 & 15 & 25 & 14 & 02 & 22 & 27 & 27 & 38 & 28 & 26 & 39 & 15 & 18 & 11 & 18 & 50 & 48 & 34 & 07 & 30 & 80 & 47 & 35 & 37 & 13 & 70 & 11 & 44 & 20 & 76 & 23 & 33 & 56 & 31\end{array}$

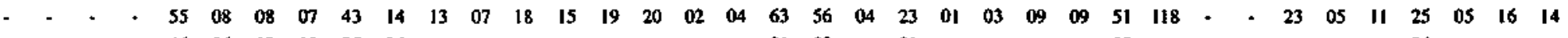

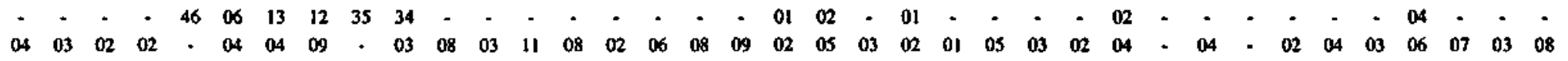
$\begin{array}{cccccccccccccccccccccccccccccccccccccc}04 & 03 & 02 & 02 & - & 04 & 04 & 09 & - & 03 & 08 & 03 & 11 & 08 & 02 & 06 & 08 & 09 & 02 & 05 & 03 & 02 & 01 & 05 & 03 & 02 & 04 & - & 04 & - & 02 & 04 & 03 & 06 & 07 & 03 & 08 \\ - & - & - & - & 43 & - & 03 & - & 22 & - & 31 & - & - & - & - & - & 01 & 43 & - & 20 & - & 09 & - & - & - & - & - & 13 & - & - & 02 & - & - & 01 & - & - & -\end{array}$

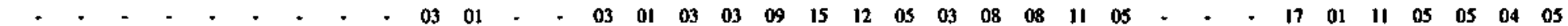

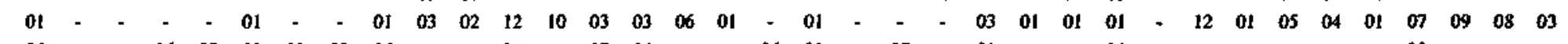

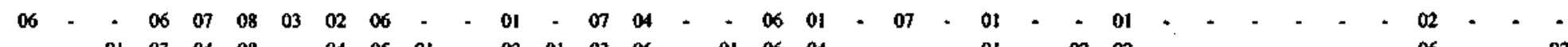

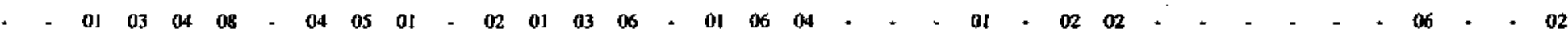

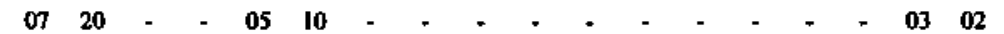

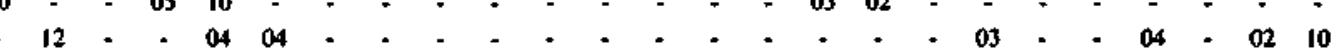

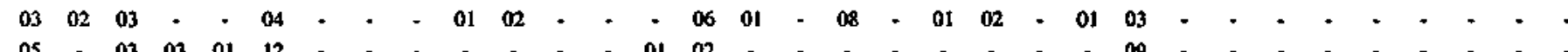
$\begin{array}{lllllll}05 & - & 03 & 03 & 01 & 12 & \text {. }\end{array}$

$02-01$ o1 $-\quad-01025$ $\therefore \quad-\quad 1606$ $\begin{array}{llllll}06 & - & 01 & 02 & 03 & 01\end{array}$ $06 \quad 04 \div 02 \quad 01 \quad 02$ $-01 \quad-06-101-02$

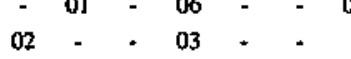

$$
\begin{array}{ccccccccc}
- & - & - & - & - & - & - & 08 & 03 \\
02 & - & - & 01 & 01 & - & - & 01 & 01
\end{array}
$$$$
\cdot .+. \cdot . \cdot 0303
$$$$
01 \cdot * \cdot-0203 \cdot 03.09 * 2.04
$$$$
1606 \cdot=
$$
01 


\begin{tabular}{|c|c|c|c|c|c|c|c|c|c|c|c|c|c|c|c|c|c|c|c|c|c|c|c|c|c|c|c|c|c|c|c|c|c|c|c|c|c|}
\hline AMOSTRAS & 01 & 02 & 03 & 04 & 05 & 06 & 07 & 08 & 09 & 10 & 11 & 12 & 13 & 14 & 15 & 16 & 17 & 18 & 19 & 20 & 21 & 24 & 27 & 28 & 32 & 33 & 34 & 35 & 38 & 41 & 45 & 47 & 49 & so & 51 & 52 & 54 \\
\hline \multicolumn{38}{|l|}{ ESPECTES } \\
\hline \multicolumn{38}{|l|}{ BENTÓNICAS } \\
\hline Pyrgo patagotica & - & - & - & - & - & - & - & - & - & - & - & - & - & - & - & - & - & . & - & $=$ & . & - & - & $=$ & - & $=$ & - & 03 & - & - & - & - & . & + & - & - & 01 \\
\hline Pyrgo nasuta & - & $=$ & - & . & - & - & - & . & . & 02 & $=$ & OI & $=$ & - & 0I & - & $\cdot$ & . & - & $=$ & * & $=$ & . & . & - & - & * & - & . & - & - & - & . & - & . & - & - \\
\hline Quinqueloculing horrida & - & - & - & - & - & - & - & - & - & $=$ & $=$ & - & - & - & - & - & - & . & 01 & 02 & - & - & . & 01 & - & - & - & - & - & - & - & - & - & - & * & - & - \\
\hline Spiroloctulina moesi & . & . & - & - & - & - & $=$ & - & - & - & - & - & - & 01 & - & - & - & * & - & 02 & al & - & - & . & . & . & $=$ & - & - & $=$ & . & . & * & $=$ & - & $=$ & - \\
\hline Siphonisa reticulata & - & - & - & . & - & - & - & - & - & - & - & - & - & - & . & - & - & - & - & $=$ & - & - & . & $=$ & - & 01 & - & - & $=$ & - & 02 & - & . & - & ol & - & - \\
\hline Quinqueloculina microcontulata & - & - & - & - & 01 & - & 01 & - & - & - & - & - & - & of & & - & - & - & - & - & . & . & . & . & . & - & - & . & - & - & . & - & - & . & - & - & - \\
\hline Quingqueloculina polygond & - & - & - & - & - & - & - & - & - & - & . & - & $=$ & - & $=$ & - & . & - & 01 & 02 & - & - & - & . & - & - & - & - & $=$ & $=$ & - & - & $=$ & - & - & - & - \\
\hline Texrularia gramem & - & - & - & - & 01 & - & - & - & - & - & $=$ & - & - & - & - & - & - & - & - & - & - & - & - & - & - & - & 02 & - & - & - & - & $=$ & - & - & $=$ & - & - \\
\hline Ammonia beccaril & . & - & - & - & $\cdot$ & - & - & - & $\cdot$ & 02 & - & - & - & - & - & - & - & . & - & - & . & $*$ & . & . & - & $=$ & $\cdot$ & - & $\cdot$ & $\cdot$ & - & $\cdot$ & . & $\cdot$ & - & + & . \\
\hline Comusping involivens & $\cdot$ & - & $*$ & - & - & - & - & - & - & - & - & - & - & 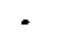 & $=$ & $=$ & $\cdot$ & . & - & 02 & - & - & . & . & + & - & - & - & - & - & - & . & . & - & . & . & . \\
\hline Florilus graseloupi & $\cdot$ & $\cdot$ & $\cdot$ & - & $\cdot$ & $\cdot$ & - & - & $=$ & - & - & - & $\cdot$ & $\cdot$ & $=$ & $\cdot$ & - & - & $\cdot$ & 02 & $\cdot$ & - & - & - & - & 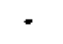 & - & $=$ & $=$ & - & $\cdot$ & $=$ & $=$ & - & $=$ & * & - \\
\hline Hanzawaia berthelott & - & - & - & - & - & - & - & - & 01 & - & - & - & - & - & - & - & - & - & * & - & - & 01 & - & - & * & - & - & - & - & - & - & - & - & + & - & + & - \\
\hline Miliotinello labiosa & - & - & - & . & - & - & - & - & - & - & $=$ & $=$ & - & - & - & - & - & - & - & - & - & - & 01 & - & * & - & - & 02 & - & - & - & - & - & - & - & - & - \\
\hline Pyrgo bullotidst & - & $\cdot$ & - & - & - & - & - & 02 & - & - & - & - & - & - & - & - & - & - & - & - & - & - & - & - & - & - & - & $\cdot$ & - & - & - & - & - & - & - & - & - \\
\hline Pyrgo Lafmanersts & - & of & - & - & . & - & - & - & - & - & $\cdot$ & - & - & - & 01 & $=$ & - & - & - & - & $\cdot$ & - & - & - & * & - & - & - & - & - & - & $=$ & $=$ & - & $=$ & - & - \\
\hline Quinqueloculina dispariltis curta & - & . & . & . & - & - & . & - & - & - & - & - & . & - & - & - & - & - & . & 02 & - & . & - & . & . & - & - & - & - & - & - & - & - & - & - & . & + \\
\hline Quingueloculina mericulata & - & - & - & - & - & - & - & - & - & - & - & - & - & . & - & - & - & - & - & - & - & - & - & - & - & - & - & - & - & - & 02 & - & . & - & - & - & $=$ \\
\hline Spirolocminat estebant & - & . & - & - & 01 & - & - & - & - & - & - & - & - & - & - & - & . & 01 & - & - & - & - & - & - & - & - & - & - & - & - & 02 & $=$ & - & - & - & - & - \\
\hline Spiroloculina profurda & - & - & - & - & $=$ & - & $=$ & - & - & $=$ & - & - & . & - & = & - & - & - & - & - & - & - & - & - & - & - & - & - & - & - & 01 & - & - & 01 & $=$ & - & - \\
\hline Textularia andeiana & - & - & - & - & - & - & - & - & - & - & $=$ & - & - & - & - & - & $=$ & - & - & - & . & - & - & - & - & . & - & - & - & - & 01 & - & = & 01 & $=$ & - & - \\
\hline Arriculina mucronata & - & - & . & - & - & - & - & $\therefore$ & - & - & - & - & - & - & - & - & - & . & - & - & - & - & - & - & - & - & - & - & - & - & 01 & - & - & - & - & - & - \\
\hline Brizalina striatula & - & - & - & - & - & - & $=$ & + & - & - & - & - & . & - & - & - & . & - & - & 02 & - & - & . & * & - & * & + & * & - & - & - & - & - & $=$ & - & $=$ & $=$ \\
\hline Ghabrarelta brasiliensis & - & - & - & - & $\mathbf{0 1}$ & - & - & - & * & - & - & * & - & - & - & - & - & DI & - & - & - & - & - & - & - & - & - & 01 & . & - & - & $=$ & - & - & - & - & - \\
\hline Miliolinella subroturda & - & - & - & - & - & - & - & - & $=$ & - & $=$ & - & - & - & - & - & - & - & 01 & - & - & - & - & - & - & - & - & - & - & - & - & - & - & - & - & - & - \\
\hline Nonionelta aflanticos & . & - & - & - & - & * & - & - & $=$ & $=$ & - & - & - & - & - & . & - & - & - & 01 & - & - & $\cdot$ & . & - & - & - & - & - & - & - & - & + & - & - & * & - \\
\hline Pullenia subcarinata quinqueloba & - & - & - & - & - & - & - & - & - & - & - & - & - & - & - & - & - & - & 01 & - & - & - & - & - & - & . & - & - & - & * & - & - & - & - & - & - & - \\
\hline Siphogenterina raphanus & - & - & * & - & - & - & - & - & - & - & - & - & - & - & - & - & - & $\therefore$ & - & 01 & - & - & - & - & - & - & $=$ & - & $=$ & $=$ & - & - & - & - & - & - & - \\
\hline Tribculita gracilis & - & . & - & - & - & . & - & - & $=$ & . & - & - & . & - & - & - & - & - & . & 01 & - & - & - & - & - & - & - & - & - & - & - & - & - & - & - & - & - \\
\hline Triloculing Iricarinota & - & - & $\cdot$ & $\cdot$ & - & $\cdot$ & $\cdot$ & $\cdot$ & $\cdot$ & $\cdot$ & - & * & $\cdot$ & & . & $\cdot$ & * & $\cdot$ & - & 01 & - & $\cdot$ & * & $\cdot$ & $\cdot$ & - & - & - & - & - & $=$ & - & $=$ & $\cdot$ & - & $=$ & - \\
\hline \multicolumn{38}{|l|}{ PLANCTONICAS } \\
\hline Globigeringldes urilobus & - & - & . & - & 05 & ol & - & - & o! & - & - & - & - & - & . & - & - & . & 08 & 05 & - & 01 & . & - & 02 & - & - & - & - & - & . & - & - & - & - & - & - \\
\hline Globigetrinoides ruber & - & 04 & - & - & . & - & - & - & - & 02 & - & - & - & - & - & . & - & - & - & - & - & - & - & - & - & - & * & - & - & - & ot & - & - & • & 03 & - & + \\
\hline Olabopotalin menardit & - & . & - & . & 02 & - & - & - & - & 02 & * & . & . & - & - & - & - & - & - & - & $\cdot$ & 01 & - & - & - & $=$ & - & 02 & - & - & 01 & - & $=$ & - & - & $\mathbf{0}$ & - \\
\hline Globigerinoides aequilateralis & - & - & - & - & - & - & - & - & - & - & - & - & - & - & 01 & - & - & - & - & - & - & - & - & - & . & - & - & 01 & - & - & - & - & - & - & - & - & - \\
\hline Globigerina bulloider & - & - & - & . & - & - & - & - & . & . & . & . & - & - & 0I & - & $=$ & - & - & - & - & . & - & - & - & - & 01 & - & $=$ & $=$ & - & - & - & $=$ & $=$ & - & - \\
\hline
\end{tabular}


Tabela 2 - Principais espécies de foraminíferos do Atol das Rocas (coleta em abril de 1991)

Table 2 - Major foraminifera species from Atol das Rocas (samples collected in April -1991)

\begin{tabular}{|l|c|c|}
\hline \multicolumn{1}{|c|}{ Esptctes } & $\begin{array}{c}\text { Frequencia } \\
\text { Absoluta }\end{array}$ & $\begin{array}{c}\text { Frequencia } \\
\%\end{array}$ \\
\hline Archaias angulatus & 7.088 & 69 \\
Amphistegina Lessonil & 1.114 & 11 \\
Soriles marginalls & 669 & 7 \\
Trilaculina subrotunda & 156 & 2 \\
Heterostegina suborbicularis & 150 & 1 \\
Triloculina trigonula & 145 & 1 \\
Borelis pulchral38 & 1 & \\
Gypsina vesicularis & 99 & 1 \\
Tritaculine neticulata f. carlinata & 62 & 1 \\
Outros & 564 & 5 \\
Total da Espleies & 10.254 & 100 \\
\hline
\end{tabular}

Tabela 3 - Número de foraminíferos mortos/vivos por amostra

Table 3 - Number of dead/alive foraminifera per sample

\begin{tabular}{|c|c|c|c|}
\hline Amostras & $\begin{array}{l}\text { Número de } \\
\text { Foraminifferos } \\
\text { Mortos }\end{array}$ & $\begin{array}{l}\text { Nómero de } \\
\text { Foraminfferos } \\
\text { Vivos }\end{array}$ & $\begin{array}{c}\text { Relaç5o } \\
\mathrm{M} / \mathrm{V}\end{array}$ \\
\hline 01 & 297 & 03 & $99 / 1$ \\
\hline 02 & 298 & 02 & $149 / 1$ \\
\hline 03 & 297 & 03 & $99 / 1$ \\
\hline 04 & 288 & 12 & $24 / 1$ \\
\hline 05 & 296 & 04 & $74 / 1$ \\
\hline 06 & 297 & 03 & $99 / 1$ \\
\hline 07 & 298 & 02 & $149 / 1$ \\
\hline 08 & 300 & $\infty$ & $300 / 0$ \\
\hline 09 & 297 & 03 & $99 / 1$ \\
\hline 10 & 296 & 04 & $74 / 1$ \\
\hline 11 & 296 & 04 & $74 / 1$ \\
\hline 12 & 300 & 00 & $300 / 0$ \\
\hline 13 & 300 & 00 & $300 / 0$ \\
\hline 14 & 296 & 04 & $74 / 1$ \\
\hline 15 & 295 & 05 & $59 / 1$ \\
\hline 16 & 295 & os & $59 / 1$ \\
\hline 17 & 300 & 00 & $300 / 0$ \\
\hline 18 & 300 & $\infty$ & $300 / 0$ \\
\hline 19 & 299 & 01 & $299 / 1$ \\
\hline 20 & 300 & 00 & $300 / 0$ \\
\hline 21 & 300 & $\infty 0$ & $300 / 0$ \\
\hline 24 & 300 & $\infty$ & $300 / 0$ \\
\hline 27 & 300 & 00 & $300 / 0$ \\
\hline 28 & 300 & 00 & $300 / 0$ \\
\hline 32 & 298 & 02 & $149 / 1$ \\
\hline 33 & 300 & $\infty$ & $300 \%$ \\
\hline 34 & 300 & $\infty 0$ & $300 / 0$ \\
\hline 35 & 288 & 12 & $24 / 1$ \\
\hline 38 & 208 & 00 & $208 / 0$ \\
\hline 41 & 16 & 00 & $16 / 0$ \\
\hline 45 & 297 & 03 & $99 / 1$ \\
\hline 47 & 300 & 00 & 30010 \\
\hline 50 & 300 & $\infty$ & $300 / 0$ \\
\hline 51 & 298 & 02 & $149 / 1$ \\
\hline 52 & 300 & $\infty$ & $300 / 0$ \\
\hline
\end{tabular}

Tabela 4 -Distribuição do número de espécies por amostra Table 4 - Distribution of the number of species per sample

\begin{tabular}{|c|c|c|}
\hline Localiziplo & Amostas & $\begin{array}{c}N^{*} \text { de } \\
\text { Espeties }\end{array}$ \\
\hline $\begin{array}{l}\text { Depdsito Arenoso (32 esptcies) } \\
\text { lagune sempre submerst - } 5 \mathrm{~m} \\
\text { parte com submersto } \\
\text { periodien - } 40 \mathrm{~cm}\end{array}$ & $\begin{array}{c}11-12-13-14-18 \\
6-24-16-17 \text { (metade oetle) } \\
32-33-34-35-51 \text { (metade leste) }\end{array}$ & $\begin{array}{l}18 \\
25 \\
16\end{array}$ \\
\hline egtrada de Bartetinha $.60 \mathrm{~cm}$ & $1-3-5-7-9$ & 37 \\
\hline $\begin{array}{l}\text { Piscinas do Topa Recifol } \\
\text { (45 expeciet) } 1,5 \mathrm{~m}\end{array}$ & $\begin{array}{l}19-20 \text { (a oeste) } \\
45-52 \text { (a leste) }\end{array}$ & $\begin{array}{l}40 \\
14\end{array}$ \\
\hline $\begin{array}{l}\text { Topo Recifil (25 esptcies) } 40 \mathrm{em} \\
\text { com submersto pertica }\end{array}$ & $\begin{array}{l}2-15 \text { (a nordeste) } \\
21 \text { (a sudoeste) } \\
50 \text { (a leste) }\end{array}$ & $\begin{array}{c}18 \\
8 \\
13\end{array}$ \\
\hline Ilha do farol (19 explcies) $50 \mathrm{~cm}$ & $4-8-47$ & 19 \\
\hline
\end{tabular}

Peneroplis pertusus, Triloculina trigonula, Dentostomina enoplostoma, Discorbis mira, Elphidium galvestonensis, Hanzawaia bertheloti, Pyrgo nasuta, Spiroloculina profunda, Textularia gramem e Globorotalia menardii só ocorrem a oeste, enquanto que Siphonina reticulaia, Textularia candeiana e Globigerina bulloides só ocorrem a leste da laguna.

Na piscina do topo do recife (Tabela 6), das 45 espécies isoladas, 14 são encontradas a leste da área e, apenas 4, são

Tabela 5-Distribuição dos foraminíferos no depósito arenoso - zona com submersão periódica

Table 5 - Distribution of foraminifera in the sandy deposit - periodically submersed area

\begin{tabular}{|c|c|c|}
\hline Amostras & Metade Oeste & Metade Leste \\
\hline Amphistegina lessonili & $6-26-16-17$ & $32-34-51$ \\
\hline Archaias angularus & $6-24-16-17$ & $32-34-51$ \\
\hline Heterostegina suborbicularis & $6-24-16-17$ & $32-34 \cdot 51$ \\
\hline Peneroplis bradyi & $6-16$ & * \\
\hline Peneroplis discoldeus & $6-24$ & - \\
\hline Peneroplis pertusus & 6 & . \\
\hline Siphonina reticulara & - & 51 \\
\hline Sorites marginalis & $6-24-16-17$ & $32-34.51$ \\
\hline Trilacutina subrolunda & $6-24$ & 32 \\
\hline Triloculina reficulata f carinata & $6-24-16$ & 34 \\
\hline Trilaculina reficulata f. bicarinata & 6 & 32-34 \\
\hline Triloculino reticulata f typica & $6-24-16-17$ & 34 \\
\hline Trilaculina quadrilateralis & 24 & $32-34-51$ \\
\hline Trilaculina trigonula & 24 & - \\
\hline Globigerina bulloides & $=$ & 34 \\
\hline Borells pulchra & 24-16-17 & $32-51$ \\
\hline Dentostomina enoplostoma & 24 & - \\
\hline Discorbit mira & 16 & 32 \\
\hline Elphidium galvestonensis & 24 & - \\
\hline Gipsina vesicularis & 24-16-17 & $32-34-51$ \\
\hline Hanzawaia berthelori & 24 & - \\
\hline Pyrgo nasula & 24 & - \\
\hline Spiroloculina profunda & 16 & - \\
\hline Textularia gramen & 16 & - \\
\hline Texularia candeiana & - & 32 \\
\hline Globigerinoides trilobus & $6-24$ & 32 \\
\hline Globorolalia menardii & 24 & - \\
\hline
\end{tabular}




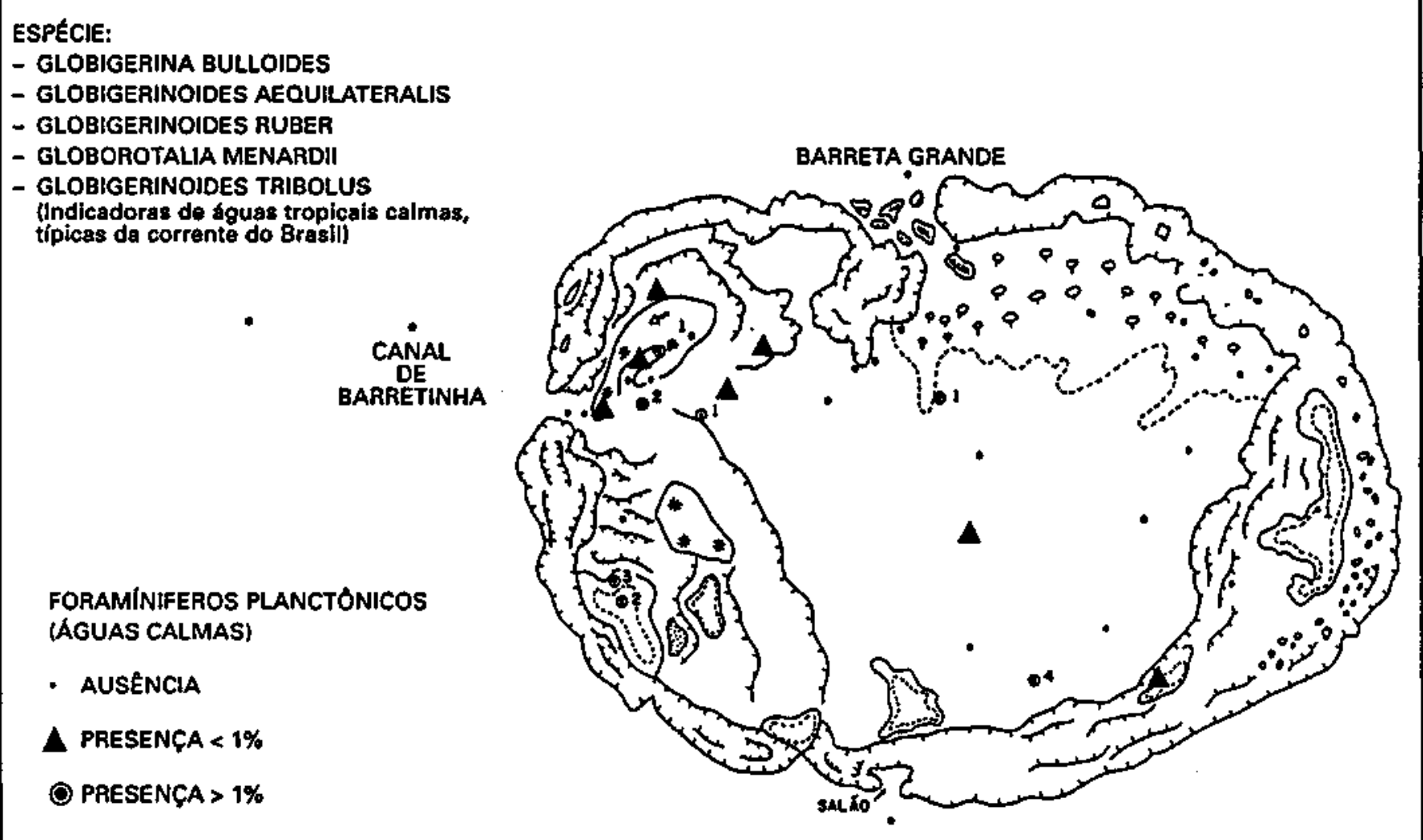

Figura 4 -Distribuicão das especies planciónicas

Figure 4-Distribution of planetonics specie:

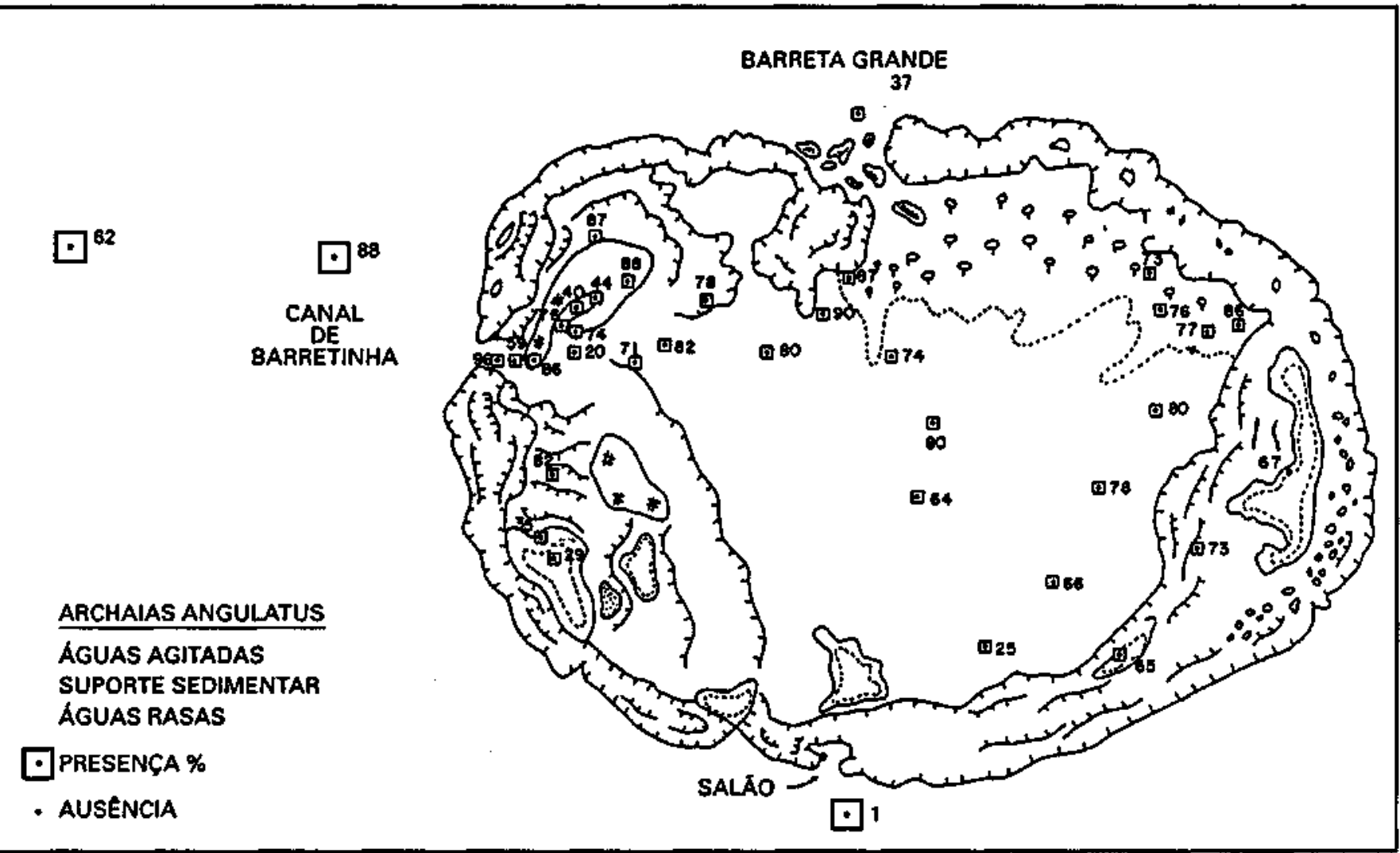

Figura 5 -Distribuição de Archaias angulatus e o seu significado ecológico Figure 5 - Distribution of Archaias angulatus and its ecologic significance 


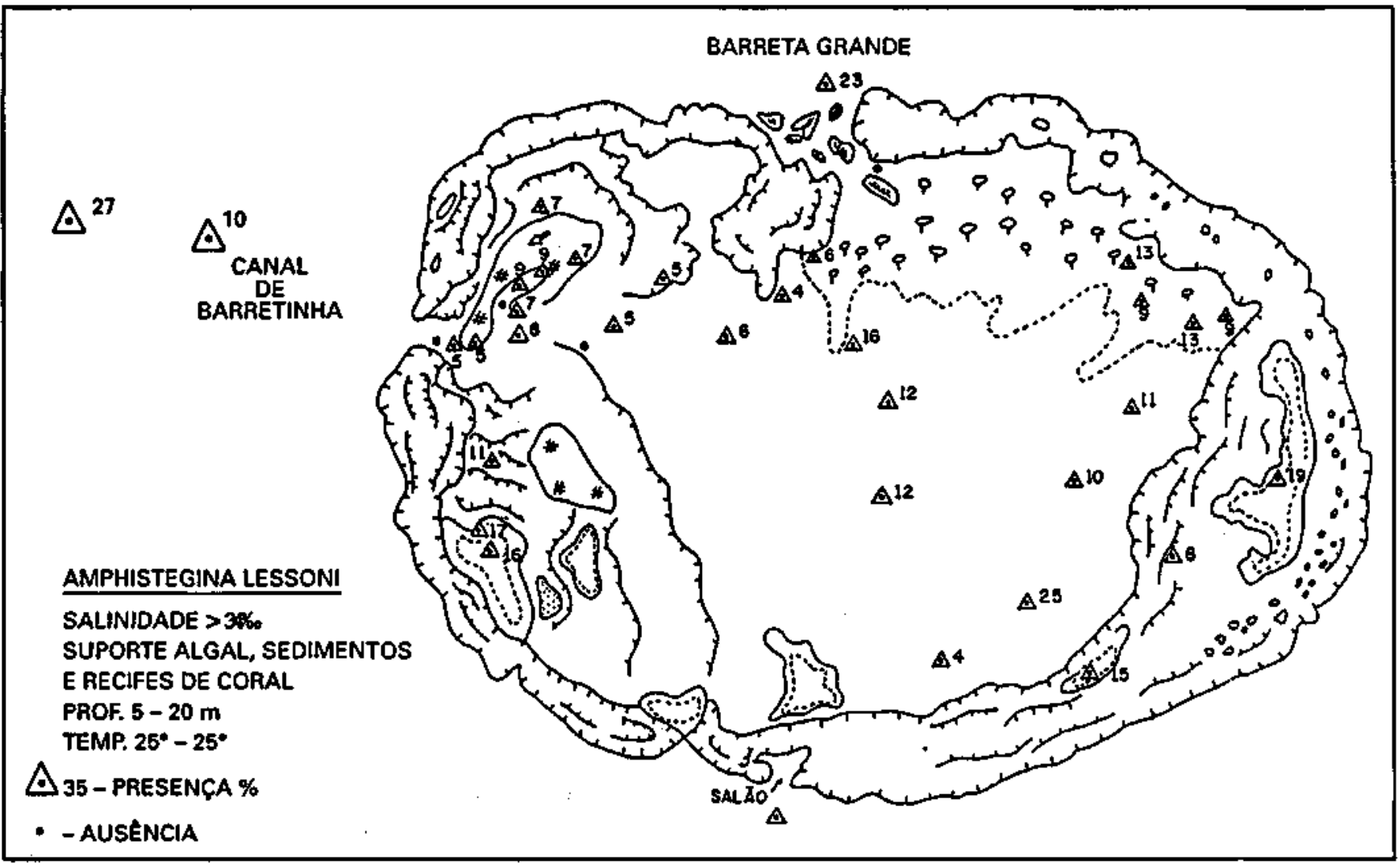

Figura 6 - Distribuição de Amphistegina lessonii e o seu significado ecológico Figure 6 - Distribution of Amphistegina lessonii and its ecologic significance

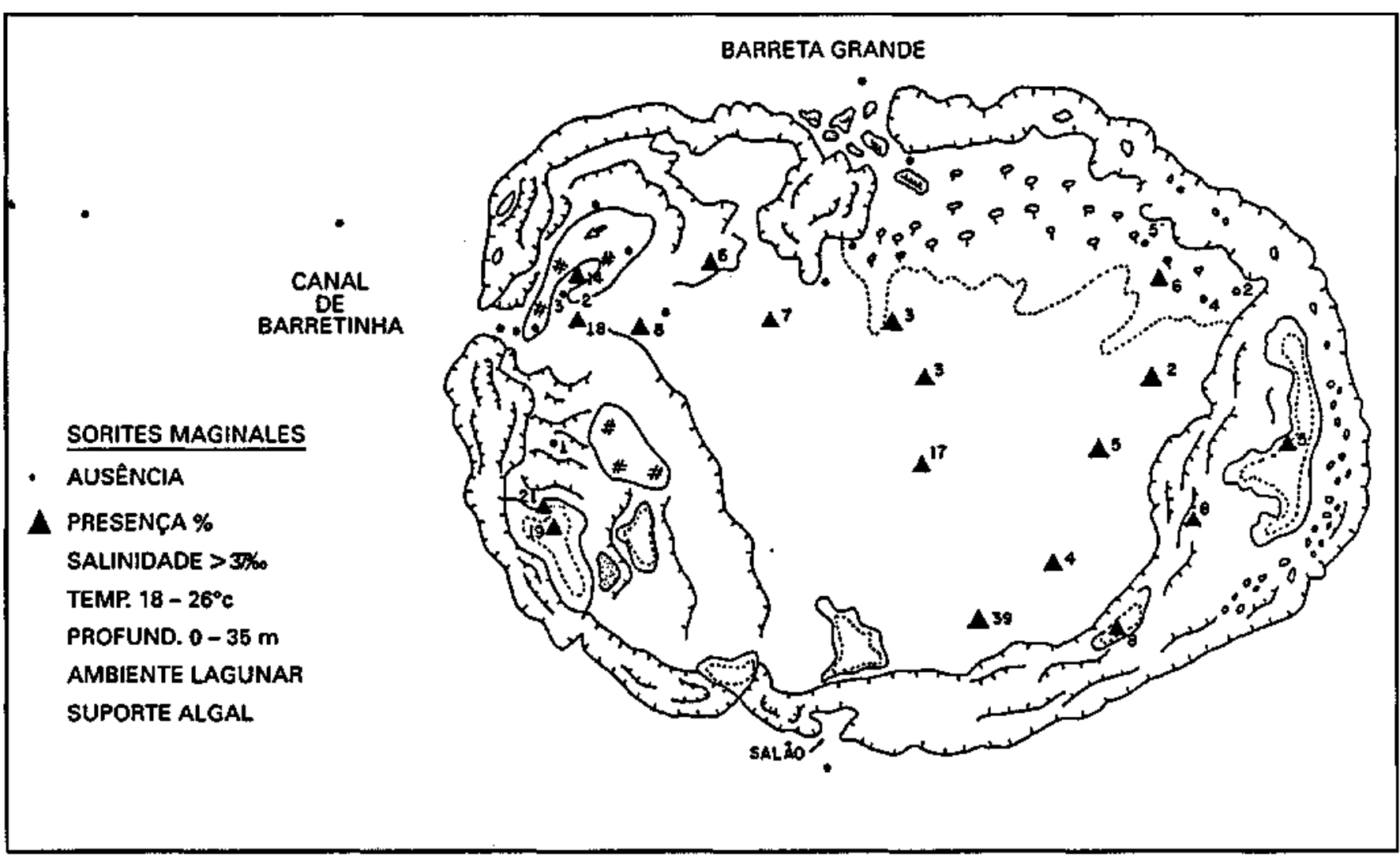

Figura 7 - Distribuição de Sorites marginalis e o seu significado ecológico Figure 7 - Distribution of Sorites marginalis and its ecologic significance 
exclusivas Quinqueloculina reticulata, Siphonina reticulata, Textularia agglutinanse Globigerinoides ruber. Siphonina pulchra não foi considerada exclusiva por estar presente na parte oeste do topo recifal.

Nas amostras do topo do recife com submersão periódica (Tabela 7), este fato se repete em relação às espécies do gênero Peneroplis e da espécie Discorbis mira, no lado oeste. Triloculina trigonula ocorre apenas nas amostras a leste do anel recifal. Devido às características fisiográficas do topo recifal, outras espécies compõem a fauna exclusiva da parte oeste: Pyrgo tainanensis, Siphonina pulchra, Spiroloculina estebani, Triloculina elongata, Triloculina reticulata $f$. bicarinata, Globigerinoides aequilateralis e Globigerinoides ruber. As espécies Spiroloculina profunda e Trethompalus bulloides constituem, juntamente com Triloculina trigonula, já mencionada, a microfauna leste do Atol. A espécie Triloculina subrotunda não foi considerada por estar presente a oeste da ilha do Farol.

Tabela 6 - Distribuição dos foraminíferos nas areias das piscinas e do topo recifal

Table 6- Distribution of foraminifera in the sandy deposit of the pools and reef top

\begin{tabular}{|c|c|c|}
\hline Amostras & Parte Oeste & Parte Leste \\
\hline 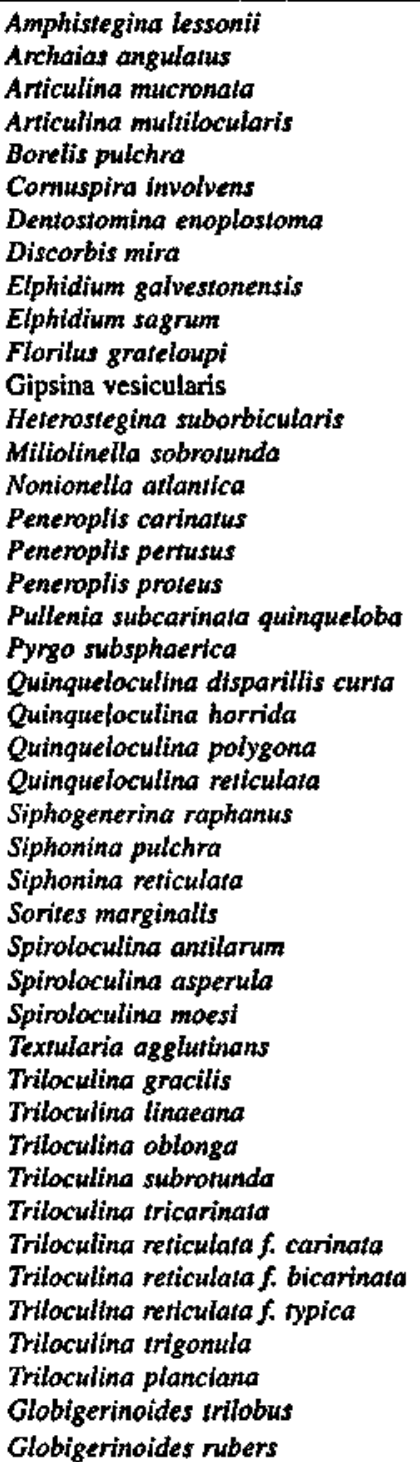 & $\begin{array}{c}19-20 \\
19-20 \\
20 \\
20 \\
19-20 \\
20 \\
19-20 \\
19-20 \\
20 \\
19 \\
20 \\
20 \\
19-20 \\
19 \\
20 \\
19-20 \\
19-20 \\
19-20 \\
19 \\
19-20 \\
20 \\
19-20 \\
19-20 \\
= \\
20 \\
= \\
2 \\
19-20 \\
19-20 \\
19 \\
20 \\
2 \\
20 \\
20 \\
19-20 \\
19-20 \\
19-20 \\
19-20 \\
19-20 \\
19-20 \\
19-20 \\
19 \\
= \\
19-20 \\
20\end{array}$ & 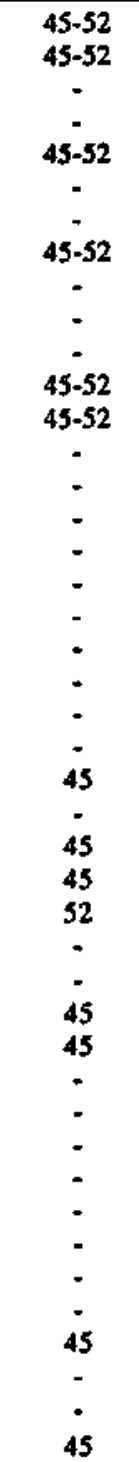 \\
\hline
\end{tabular}

Tabela 7-Distribuição dos foraminíferos no topo recifal (submersão periódica)

Table 7 - Distribution of foraminifera on the reef top (periodically submersed)

\begin{tabular}{|c|c|c|c|}
\hline Amostras & $\mathbf{N} / \mathbf{w}$ & $\mathbf{W}$ & Lesie \\
\hline Amphistegina lessonit & 2-15 & 21 & 50 \\
\hline Archaias angulatus & $2-15$ & 21 & 50 \\
\hline Articulina multilocularis & $=$ & 21 & 50 \\
\hline Borelis pulchra & 15 & 21 & so \\
\hline Discorbils mira & 15 & - & - \\
\hline Gipsina vesicularis & 2-15 & $=$ & 50 \\
\hline Globlgerinoides aequilateralis & 15 & $=$ & $=$ \\
\hline Globigerinoides ruber & 2 & - & $=$ \\
\hline Heterostegina subarbicularis & 2-15 & 21 & 50 \\
\hline Peneroplis bradyl & 15 & - & - \\
\hline Peneroplis discoideus & 2-15 & - & $\cdot$ \\
\hline Peneroplis pertusus & 2 & $=$ & - \\
\hline Pyrgo subsphaerica & $2-15$ & * & 50 \\
\hline Pyro tainanensis & 2-15 & • & $=$ \\
\hline Siphonina pulchra & $=$ & 21 & $=$ \\
\hline Sorites marginalis & 15 & 21 & 50 \\
\hline Spiroloctulina estabant & - & 21 & - \\
\hline Spiroloculina profunda & $=$ & - & 50 \\
\hline Tretomphalus bulloides & - & - & 50 \\
\hline Trilocutina elongaia & 15 & - & - \\
\hline Thiloculina rellculata f. carinata & $2-15$ & - & 50 \\
\hline Triloctina reficulara f. bicarinala & 15 & - & - \\
\hline Triloculina reticulala f. topica & 15 & - & 50 \\
\hline Triloculina sobrotunda & - & - & 50 \\
\hline Triloculina trigonula & - & . & so \\
\hline
\end{tabular}

Variação da microfauna nos subambientes Comparando-se as listas das espécies de foraminíferos nos diversos subambiente observa-se que a distribuição das espécies acompanha essas variações do ambiente. As espécies são, na sua maioria, raras e pouco representativas e podem ser agrupadas da seguinte forma: a) espécies adaptadas a laguna (zona com submersão permanente) (Tabela 8); b) especies adaptadas ao depósito arenoso (Tabela 5); c) espécies adaptadas às piscinas do topo recifal (Tabela 6); d) espécies que independem da localização no depósito e laguna, mas ausentes nas piscinas e, e) espécies indiferentes às variações do ambiente (Tabela 9).

Na ilha do Farol, das 19 espécies encontradas (Tabela 10), somente Elphidium sagrum e Pyrgo bulloides não se encontram nas piscinas e depósitos arenosos.

$\mathrm{Na}$ entrada de Barretinha a microfauna de foraminíferos corresponde ao depósito arenoso, com exceção de 9 espécies, assinaladas com asterisco na Tabela 11. Vale ressaltar que elas estão presentes somente nas amostras 05 e 09 .

Relação Miliolina/Rotalina/Textulariina $\mathrm{O}$ estudo da frequêencia das assembléias de foraminíferos Miliolina, Rotaliina e Textulariina (Tabela 12) e a elaboração do diagrama triangular de Murray (1973) (Figuras 3 e 3a) indicam a abundância das duas primeiras assembléias. Este resultado reflete a existência de um ambiente marinho normal a hipersalino. Esta evidência é confirmada também pela presença de Sorites marginalis (Figura 5).

Diversidade dos Miliolideos (Triloculina) Os Miliolideos do gênero Triloculina chamam a atenção no Atol pela presença de 12 espécies, e entre essas, Triloculina reticulata, representada pelas variedades: typica, carinata $\mathrm{e}$ bicarinata (Tabela 13). 


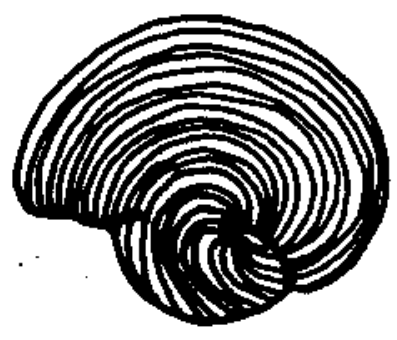

Archaias engulatus

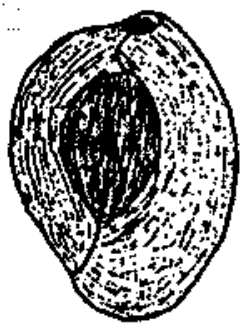

Triloculina sobrotunda

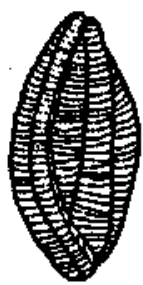

Borelis pulchra

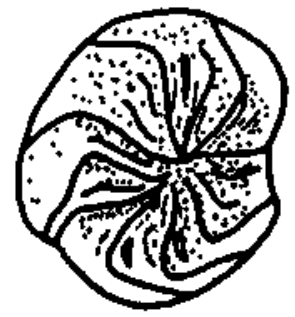

Amphistegina lessont

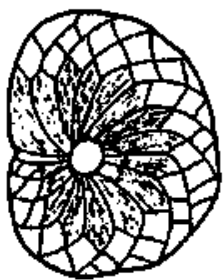

Heterostegina suborbicularis

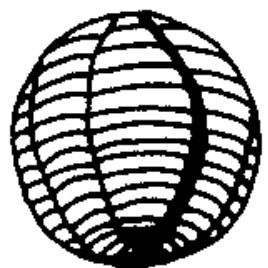

Boralis pulchra

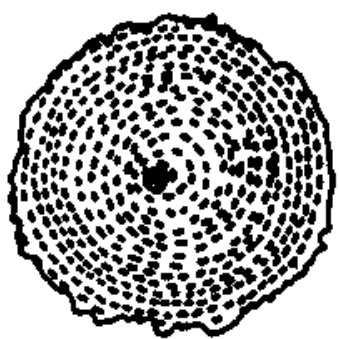

Sorites marginalis

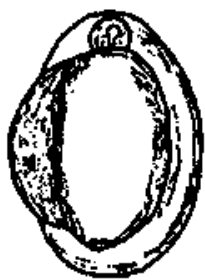

Gypsina vesicularis

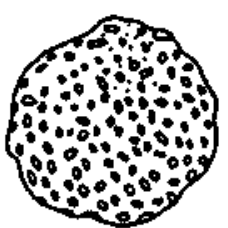

Triloculina trigonula

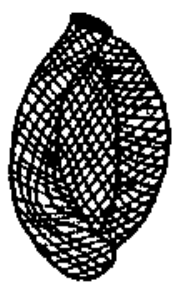

Triloculina reticulste

f. carinata

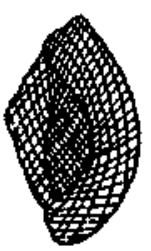

Triloculina reticulata

f. typica

Figura 8 -Distribuição dos fragmentos de colônias de bríozoáríos nos sedimentos do Atol das Rocas (freqüência absoluta) Figure 8 - Distribution of bryozoans colony fragments in the Atol das Rocas sediments (absolut frequence) 
Tabela 8 - Distribuição dos foraminíferos na laguna Table 8 - Distribution of foraminifera in the lagoon

\begin{tabular}{|c|c|}
\hline Especies & Amostras \\
\hline 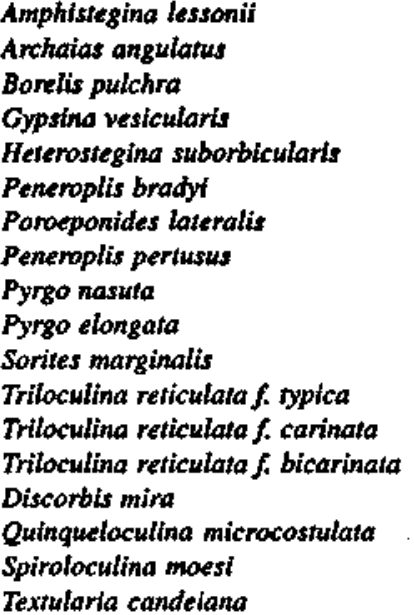 & $\begin{array}{c}11-12-13-14-18 \\
11-12-13-14-18 \\
11-12-18 \\
1]-12-13-14-18 \\
11-12-13-14-18 \\
18 \\
11-14-18 \\
14 \\
11-14 \\
18 \\
11-12-13-14-18 \\
11-13-14-18 \\
13 \\
14 \\
13 \\
14 \\
14 \\
14\end{array}$ \\
\hline
\end{tabular}

Tabela 9 - Espécies de foraminíferos lagunares com (1) ou sem (2) submersão periódica

Table 9 - Foraminifera species in the atoll lagoon with (1) or without (2) periodical submersion

\begin{tabular}{|c|c|c|c|c|}
\hline \multirow{2}{*}{\multicolumn{2}{|c|}{ Especies }} & \multicolumn{2}{|c|}{$\begin{array}{c}\text { Lacalizaçao Laguna } \\
\text { Principal }\end{array}$} & \multirow{2}{*}{\begin{tabular}{|c|}
$\begin{array}{l}\text { Lagunas } \\
\text { do Topo } \\
\text { Principal }\end{array}$ \\
3 \\
\end{tabular}} \\
\hline & & 1 & 2 & \\
\hline a) & $\begin{array}{l}\text { Pyrgo elongata } \\
\text { Poroponides lateralis } \\
\text { Quingueloculina microcostulasa } \\
\text { Spiroloculina moest } \\
\text { Heterostegina suborbicularis }\end{array}$ & $\begin{array}{l}+ \\
+ \\
+ \\
+ \\
+\end{array}$ & $\begin{array}{l}= \\
\dot{=} \\
= \\
=\end{array}$ & $\begin{array}{l}- \\
\dot{-} \\
\dot{ }\end{array}$ \\
\hline b) & $\begin{array}{l}\text { Penenoplis bradyi } \\
\text { Pyrga nasuta } \\
\text { Sorites marginalis } \\
\text { Textularla candelana } \\
\text { Triloculina reticulata f carinata } \\
\text { Triloculina reticulala f. bicarinata } \\
\text { Triloculina reticulata f. typica }\end{array}$ & $\begin{array}{l}+ \\
+ \\
+ \\
+ \\
+ \\
+ \\
+\end{array}$ & $\begin{array}{l}+ \\
+ \\
+ \\
+ \\
+ \\
+ \\
+\end{array}$ & $\begin{array}{l}: \\
\dot{.} \\
\dot{-} \\
-\end{array}$ \\
\hline c) & $\begin{array}{l}\text { Penemplis discoideus } \\
\text { Sphonina reticulata } \\
\text { Triloculind subrotunda } \\
\text { Triloculina quadrilateralis } \\
\text { Tritocullna trigonula } \\
\text { Globigerinoides rrilobus } \\
\text { Globonotalia menandii } \\
\text { Globigerina bulloldes } \\
\text { Hanzawaia bertheloti } \\
\text { Textularia gramem }\end{array}$ & $\begin{array}{l}- \\
- \\
: \\
: \\
: \\
- \\
=\end{array}$ & $\begin{array}{l}+ \\
+ \\
+ \\
+ \\
+ \\
+ \\
+ \\
+ \\
+ \\
+\end{array}$ & $\begin{array}{l}- \\
= \\
= \\
= \\
= \\
= \\
=\end{array}$ \\
\hline d) & $\begin{array}{l}\text { Elphidium galvestonensis } \\
\text { Penteroplis proteus } \\
\text { Dentostomina enoplostoma }\end{array}$ & $\begin{array}{l}. \\
.\end{array}$ & $\begin{array}{l}+ \\
+ \\
+\end{array}$ & $\begin{array}{l}+ \\
+ \\
+\end{array}$ \\
\hline e) & $\begin{array}{l}\text { Amphissegina lessonif } \\
\text { Anchaias angularus } \\
\text { Bonelis pulchra } \\
\text { Gypsina vesicularis } \\
\text { Milionella suborbicularis } \\
\text { Peneroplis perrusus } \\
\text { Discorbis mira }\end{array}$ & $\begin{array}{l}+ \\
+ \\
+ \\
+ \\
+ \\
+ \\
+\end{array}$ & $\begin{array}{l}+ \\
+ \\
+ \\
+ \\
+ \\
+ \\
+\end{array}$ & $\begin{array}{l}+ \\
+ \\
+ \\
+ \\
+ \\
+ \\
+\end{array}$ \\
\hline
\end{tabular}

\section{+ Presença}

- Ausência
Tabela 10-Distribuição dos foraminíferos na ilha do Farol Table 10 - Distribution of foraminifera in the Farol Island

\begin{tabular}{|c|c|}
\hline Especies & Amostras \\
\hline 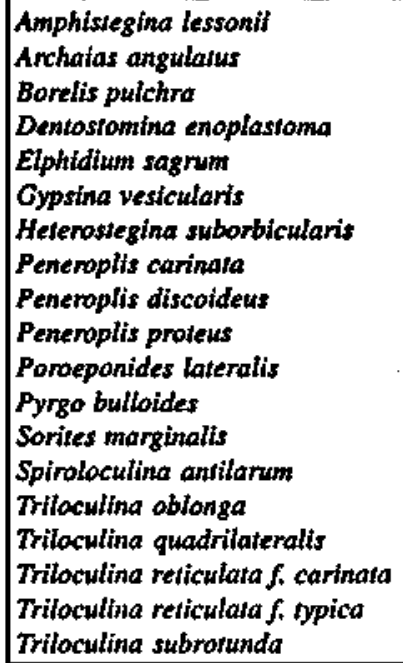 & $\begin{array}{c}4-8-47 \\
4-8-47 \\
47 \\
47 \\
4 \\
47 \\
4-8-47 \\
4-8 \\
47 \\
8 \\
8 \\
8 \\
8-47 \\
8 \\
8 \\
8 \\
8 \\
4 \\
8\end{array}$ \\
\hline
\end{tabular}

Tabela 11 - Distribuição das espécies de foraminíferos no canal de Barretinha

Table 11 - Distribution of foraminifera species within the Barretinha Channel

\begin{tabular}{|c|c|}
\hline Especies & Amostra \\
\hline 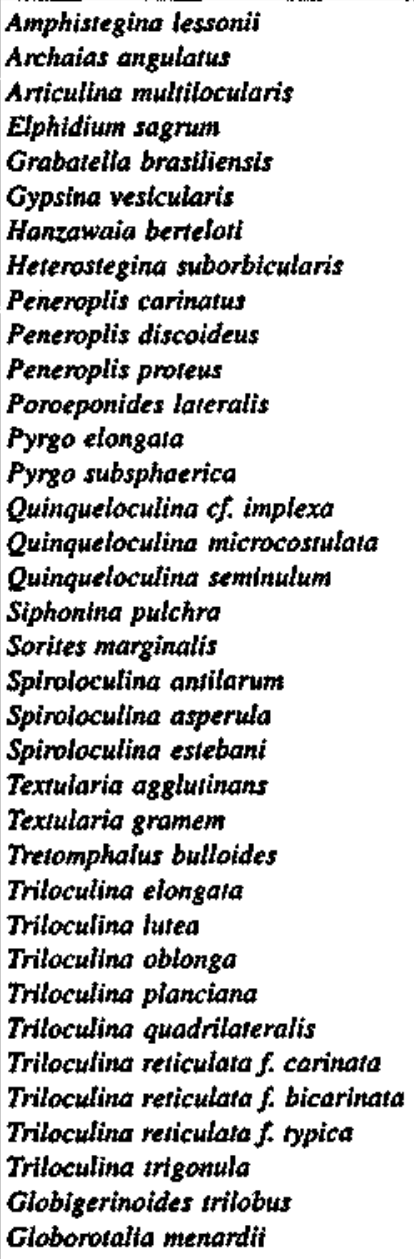 & $\begin{array}{c}1-3-5-7-9 \\
1-3-5-7-9 \\
7 \\
5 \\
5 * \\
7 \\
9 * \\
1-3-7 \\
3-7 \\
5-9 \\
5-9 \\
3-5 \\
9 \\
5 \\
9 * \\
5-7 \\
5 \\
5 \\
5-7-9 \\
5-7-9 \\
9 \\
5 * \\
5 \\
5 * \\
9 * \\
9 * \\
5 * \\
5-7-9 \\
7 \\
5-9 \\
5-7-9 \\
5-7 \\
5-7-9 \\
5-7-9 \\
5-9 \\
5 *\end{array}$ \\
\hline
\end{tabular}


Tabela 12-Percentagem das sub-ordens Rotaliina - Milioiina e Textulariina (por amostra)

Table 12 - Percentage of the sub-orders Rotaliina - Miliolina e Textulariina (per sample)

\begin{tabular}{|c|c|c|c|c|c|c|c|}
\hline Amostras & $\begin{array}{c}\text { Total } \\
\text { Espteies }\end{array}$ & $\mathbf{5}$ & $\mathbf{R}$ & $\boldsymbol{q}$ & $\mathbf{M}$ & $\%$ & $\mathbf{T}$ \\
\hline 01 & 03 & 67 & 02 & 33 & 01 & - & 00 \\
\hline 02 & 10 & 40 & 04 & 60 & 06 & $=$ & 00 \\
\hline 03 & 05 & 60 & 03 & 40 & $\alpha$ & $=$ & 00 \\
\hline 04 & 06 & 50 & 03 & 50 & 03 & - & 00 \\
\hline 05 & 25 & 28 & 07 & 64 & 16 & 8 & 02 \\
\hline 06 & 13 & 23 & 03 & 77 & 10 & $=$ & 00 \\
\hline 07 & 14 & 21 & 03 & 79 & 11 & $=$ & $\infty$ \\
\hline 08 & 13 & 23 & 03 & 77 & 10 & - & 00 \\
\hline 09 & 19 & 21 & 04 & 79 & 15 & $=$ & $\mathbf{0 0}$ \\
\hline 10 & 22 & 32 & 07 & 68 & 15 & $=$ & $\mathbf{0 0}$ \\
\hline 11 & 09 & 44 & 04 & 56 & 05 & - & 00 \\
\hline 12 & 06 & 50 & 03 & 50 & 03 & - & $\infty$ \\
\hline 13 & 08 & 50 & 04 & 50 & 04 & $=$ & $\infty 0$ \\
\hline 14 & 13 & 30 & 04 & 62 & 08 & 8 & 01 \\
\hline 15 & 16 & 31 & os & 69 & 11 & + & 00 \\
\hline 16 & 11 & 37 & 04 & 54 & 06 & 9 & 01 \\
\hline 17 & 07 & 43 & 03 & 57 & 04 & - & $\infty 0$ \\
\hline 18 & 10 & 40 & 04 & 60 & 06 & $\cdot$ & $\infty$ \\
\hline 19 & 27 & 26 & 07 & 74 & 20 & $=$ & 00 \\
\hline 20 & 35 & 29 & 10 & 69 & 24 & 2 & 01 \\
\hline 21 & 08 & 50 & 04 & 50 & 04 & - & 00 \\
\hline 24 & 21 & 29 & 06 & 71 & 15 & - & 00 \\
\hline 27 & OS & 40 & 02 & 60 & 03 & - & 00 \\
\hline 28 & 13 & 31 & 04 & 62 & 08 & 7 & 01 \\
\hline 32 & 10 & 50 & 05 & 50 & 05 & - & 00 \\
\hline 34 & 13 & 31 & 04 & 62 & 08 & 7 & 01 \\
\hline 38 & 04 & 75 & 03 & 25 & 01 & $=$ & 00 \\
\hline 41 & 03 & 67 & 02 & 33 & 01 & $=$ & 00 \\
\hline 45 & 15 & 46 & 07 & 40 & 06 & 14 & 02 \\
\hline 47 & 08 & 38 & 03 & 62 & 05 & $=$ & 00 \\
\hline 50 & 13 & 31 & 04 & 69 & 09 & * & 00 \\
\hline 51 & 08 & 50 & 04 & 50 & 04 & $=$ & 00 \\
\hline 52 & 08 & 63 & 05 & 37 & 03 & . & 00 \\
\hline
\end{tabular}

De acordo com as listas das espécies em função do ambiente, observou-se que o maior número de espécies de Triloculina ocorre na entrada de Barretinha, a oeste do depósito (amostra 05, 07 e 09), com 11 espécies e, nas piscinas do topo recifal (amostras 19 e 20), com 10 espécies. Esse número diminui para 6 e 5 espécies no topo recifal (amostras 2, 15 e 50), na parte do depósito arenoso (amostras 06, 24, 16, 17, 32, 34 e 51), e na ilha do Farol (amostra 08). A leste da laguna foram encontradas 3 espécies.

Predominam na área de estudo: Triloculina subrotunda, Triloculina trigonula, Triloculina reticulata $f$. typica $e$ Triloculina reticulata f. carinata, com freqüência relativa superior à média (7\%), muito embora a espécie mais comum seja Triloculina reticulata, que ocorre em 17 das 24 amostras, onde o gênero foi encontrado (Figura 8). É possível que as espécies estejam relacionadas não só ao tipo de substrato (areia carbonática algal), mas também às condições de estabilidade do ambiente.

BRIOZOÁRIOS NO ATOL DAS ROCAS Frequência dos fragmentos das colônias das 35 amostras analisadas, 27 continham fragmentos de colônias de briozoários, muito pequenos e, na maioria das vezes, representados apenas por um ou dois zoécios (indivíduos), dificultando inclusive a identificação na categoria de espécie. Dos 246 fragmentos isolados na totalidade das amostras estudadas, obteve-se uma freqüência média de 9 fragmentos/amostra, considerada baixa. Os valores superiores à média concentram-se nas amostras localizadas na metade leste do depósito arenoso. Vale ressaltar que apenas duas delas $\left(\mathrm{n}^{\circ *} 13\right.$ e 35) atingiram uma frequêencia absoluta superior a 30 , e elas estão associadas à sedimentos mais finos (Tabela 14, Figura 9).

A contribuição dos briozoários na composição das areias carbonáticas do Atol é muito inferior à daquela observada nas incrustações das placas (Machado et al., 1992), e isto decorre, muito provavelmente, devido à elevada fragmentação das colônias em virtude do impacto muito forte das ondas na área. As incrustações de briozoários contribuem para fixar sedimentos, manter e proteger a estrutura recifal, conforme foi assinalada por Cuffey (1972).

Principais espécies de briozoários No Atol foram isoladas 21 especies pertencentes à ordem Cheilostomata $\mathrm{e}$ 2 espécies da ordem Cyclostomata. A freqüência relativa

Tabela 13 - Distribuição dos Miliolideos (Triloculina) no Atol das Rocas (abril -1991)

Table 13 - Distribution of Miliolids (Triloculina) on the Atol das Rocas (April - 1991)

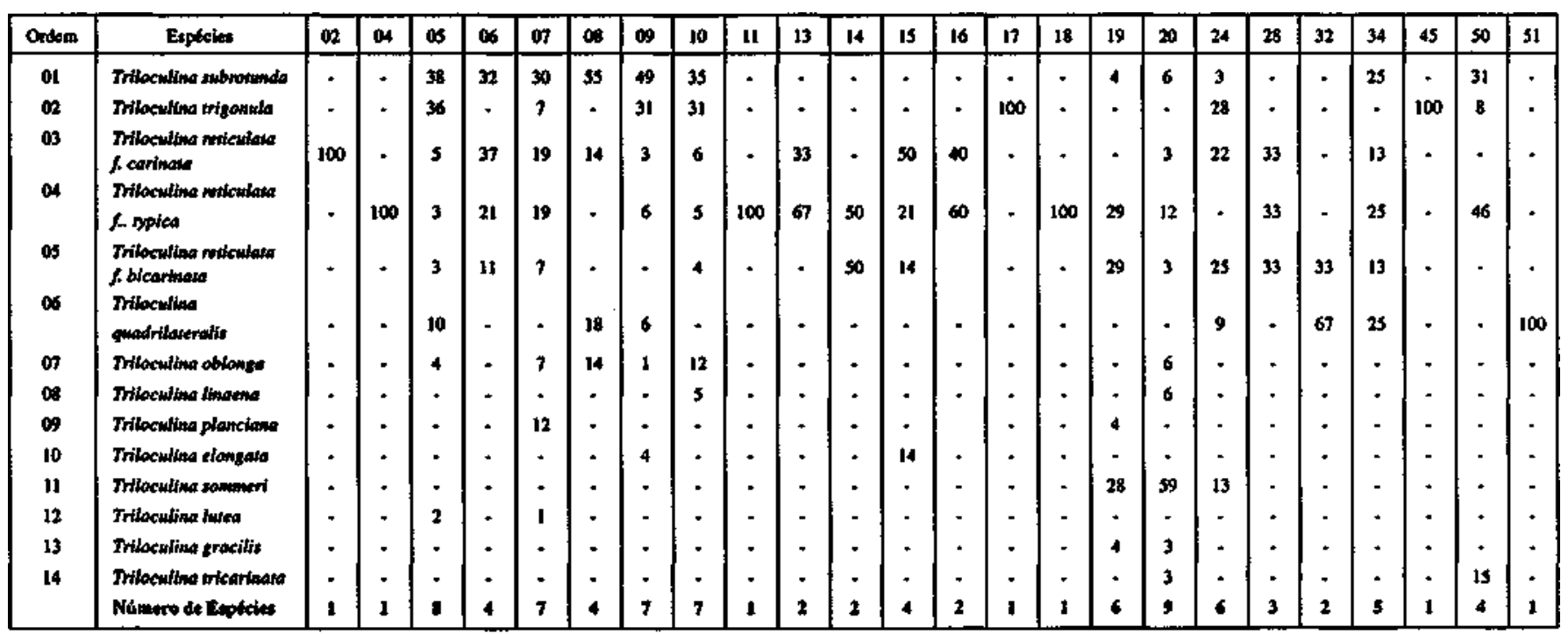




\begin{tabular}{|c|c|c|c|c|c|c|c|c|c|c|c|c|c|c|c|c|c|c|c|c|c|c|c|c|c|c|c|c|}
\hline $\begin{array}{l}\text { Amourn: } \\
\text { Eepscies }\end{array}$ & 50 & 52 & 45 & it & 13 & 14 & $5 t$ & 54 & 9 & 35 & 34 & 33 & $\$ 2$ & 39 & 44 & 16 & 15 & o6 & 46 & 19 & 20 & 02 & 05 & $\mathbf{0 3}$ & o1 & 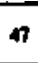 & 28 & Tolal \\
\hline Ordem Cheibutominas & & & & & & & & & & & & & & & & & & & & & & & & & & & & \\
\hline Celleporveriastsp & 3 & - & 1 & 1 & 1 & - & - & 1 & - & - & - & I & - & - & - & - & - & 1 & - & - & - & 1 & - & - & I & - & - & II \\
\hline Smintino sitanata & - & 1. & . & - & - & . & 1 & . & - & - & . & - & - & . & - & * & - & - & . & - & - & - & - & . & . & . & 1 & 3 \\
\hline 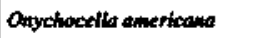 & - & 3 & . & . & - & 3 & - & - & 1 & 1 & - & I & I & - & . & - & - & - & - & - & - & - & 1 & - & - & . & - & 11 \\
\hline Solizopartits sop & . & 2 & - & - & 1 & 2 & . & 4 & - & 2 & . & 2 & 1 & 1 & - & - & . & - & - & . & - & 6 & . & I & - & . & * & $\mathbf{n 2}$ \\
\hline Trulaminparstla sp & - & 1 & - & - & 1 & $\cdot$ & $\cdot$ & $=$ & $t$ & 2 & - & • & - & - & $\mathbf{I}$ & $\cdot$ & - & $*$ & * & $\cdot$ & 1 & - & - & - & - & . & . & 7 \\
\hline Scrupacellaria sp & - & $\mathbf{I}$ & - & 1 & 4 & - & - & - & 4 & 6 & - & $\mathbf{I}$ & . & 2 & 3 & 2 & $\cdot$ & $\mathfrak{l}$ & 1 & . & 2 & $\mathbf{I}$ & 2 & $\cdot$ & - & - & - & 31 \\
\hline Repradeonellia violdicea & - & $\mathbf{I}$ & - & - & 6 & 1 & - & - & - & - & - & - & - & - & - & $\mathbf{I}$ & . & - & - & - & - & - & - & - & - & 2 & - & 11 \\
\hline Nellia doculata & - & - & $\cdot$ & 3 & 1 & 1 & - & - & $\cdot$ & $\cdot$ & $\cdot$ & $\cdot$ & - & - & - & - & $\cdot$ & - & - & $\mathbf{I}$ & - & $\cdot$ & $\mathbf{I}$ & $\cdot$ & $\cdot$ & $\cdot$ & $\mathbf{I}$ & - \\
\hline Schitopentha micotratit & $\cdot$ & $\cdot$ & . & $t$ & $\cdot$ & $\cdot$ & $\cdot$ & $\mathbf{1}$ & 1 & $\cdot$ & 1 & $\cdot$ & $\mathbf{I}$ & - & $\cdot$ & $\cdot$ & 1 & 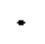 & - & 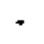 & $\cdot$ & $\cdot$ & $\cdot$ & $\cdot$ & $\cdot$ & $\cdot$ & $\cdot$ & 6 \\
\hline Amurepara noted & - & - & - & + & 1 & - & - & - & . & - & - & - & - & - & • & - & - & - & - & - & - & - & - & - & - & - & - & I \\
\hline Smintina sop & $\cdot$ & . & * & * & 1 & 1 & $\cdot$ & $\mathbf{I}$ & I & $\mathbf{I}$ & 3 & $\mathbf{I}$ & $\mathbf{I}$ & 3 & * & . & $\cdot$ & $*$ & - & . & $\cdot$ & $\mathbf{I}$ & $\mathbf{I}$ & $\cdot$ & $\cdot$ & - & - & 15 \\
\hline Ajuchosoan rastrentem + 4 & - & - & - & $\mathbf{I}$ & - & - & - & - & - & - & - & $\mathbf{I}$ & - & - & - & $\cdot$ & $\cdot$ & - & - & $\cdot$ & - & - & - & - & - & - & - & 2 \\
\hline 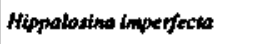 & $\cdot$ & - & . & - & 1 & - & - & - & . & $\cdot$ & - & $\cdot$ & - & . & - & $\cdot$ & - & $\cdot$ & * & - & $\cdot$ & $\cdot$ & - & - & $\cdot$ & $\cdot$ & $\cdot$ & 1 \\
\hline Thicellarin of & - & . & . & - & - & I & - & - & - & I & - & - & - & $=$ & . & . & - & - & - & - & . & . & $=$ & - & - & - & ! & F \\
\hline Strginopore la of & - & - & - & - & - & - & 1 & - & 1 & . & - & - & - & - & - & - & - & - & - & $=$ & - & - & . & - & - & - & - & 2 \\
\hline Conspertin tp & - & . & - & - & - & * & $\mathbf{l}$ & - & - & - & - & - & - & - & - & - & . & - & - & + & $\cdot$ & $\cdot$ & - & + & . & - & . & 1 \\
\hline Creidorchaema porcolons & - & - & - & - & - & - & - & + & - & 1 & - & 2 & $\cdot$ & . & $\cdot$ & $\cdot$ & - & - & - & - & - & $\mathbf{I}$ & $\cdot$ & $\cdot$ & - & - & - & 4 \\
\hline Mrrrarobdolotes op & - & $\cdot$ & $\cdot$ & - & - & $\cdot$ & - & - & - & $\cdot$ & - & $\cdot$ & $\cdot$ & $t$ & $\cdot$ & $\cdot$ & - & - & - & - & - & $\cdot$ & $\cdot$ & $\cdot$ & - & - & - & 1 \\
\hline Margaretia Ip & $\cdot$ & $\cdot$ & $\cdot$ & - & $\cdot$ & - & - & - & - & - & - & - & $\cdot$ & - & $\cdot$ & $\cdot$ & $\cdot$ & - & $\mathbf{I}$ & - & - & $\cdot$ & $\cdot$ & $\mathbf{I}$ & - & - & - & $*$ \\
\hline Crobrilarria raditiana & - & - & * & $\cdot$ & - & $\cdot$ & - & - & - & $\cdot$ & 1 & - & 1 & - & - & $\cdot$ & - & - & - & - & . & 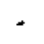 & - & - & - & - & + & 1 \\
\hline Escharine pesasterit & . & + & - & - & - & + & + & - & - & - & 1 & - & - & - & - & $\cdot$ & . & - & * & - & $\cdot$ & $\cdot$ & $\cdot$ & $\cdot$ & $\cdot$ & - & - & 1 \\
\hline N3o identiricados & 0 & 3 & 0 & 1 & 3 & 1 & 2 & 2 & $s$ & 3 & 1 & 2 & 2 & 0 & 1 & 0 & 0 & 0 & 0 & 0 & 0 & 3 & 1 & 0 & $\mathbf{1}$ & 0 & 0 & 31 \\
\hline Sub-Total & 3 & 12 & I & 6 & 21 & 10 & 4 & 9 & is & 16 & 7 & 10 & 9 & 7 & 6 & 3 & I & $\mathbf{3}$ & I & I & $\mathbf{3}$ & 14 & 1 & I & 2 & 1 & $\mathbf{3}$ & 176 \\
\hline Orden Cychostomin & 0 & 7 & 0 & 3 & 11 & 1 & 0 & 1 & 1 & 32 & 4 & 0 & 1 & 0 & 3 & 0 & 0 & 0 & 0 & 0 & 2 & 2 & 1 & 0 & 0 & 0 & o & 6 \\
\hline 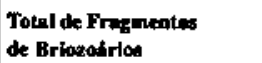 & 3 & 19 & 1 & 9 & 32 & 11 & 4 & 10 & 16 & 48 & t1 & 10 & 14 & 7 & , & 3 & 1 & 3 & 1 & 1 & $s$ & IG & $*$ & 1 & 2 & 1 & 3 & $\mathbf{w s}$ \\
\hline
\end{tabular}




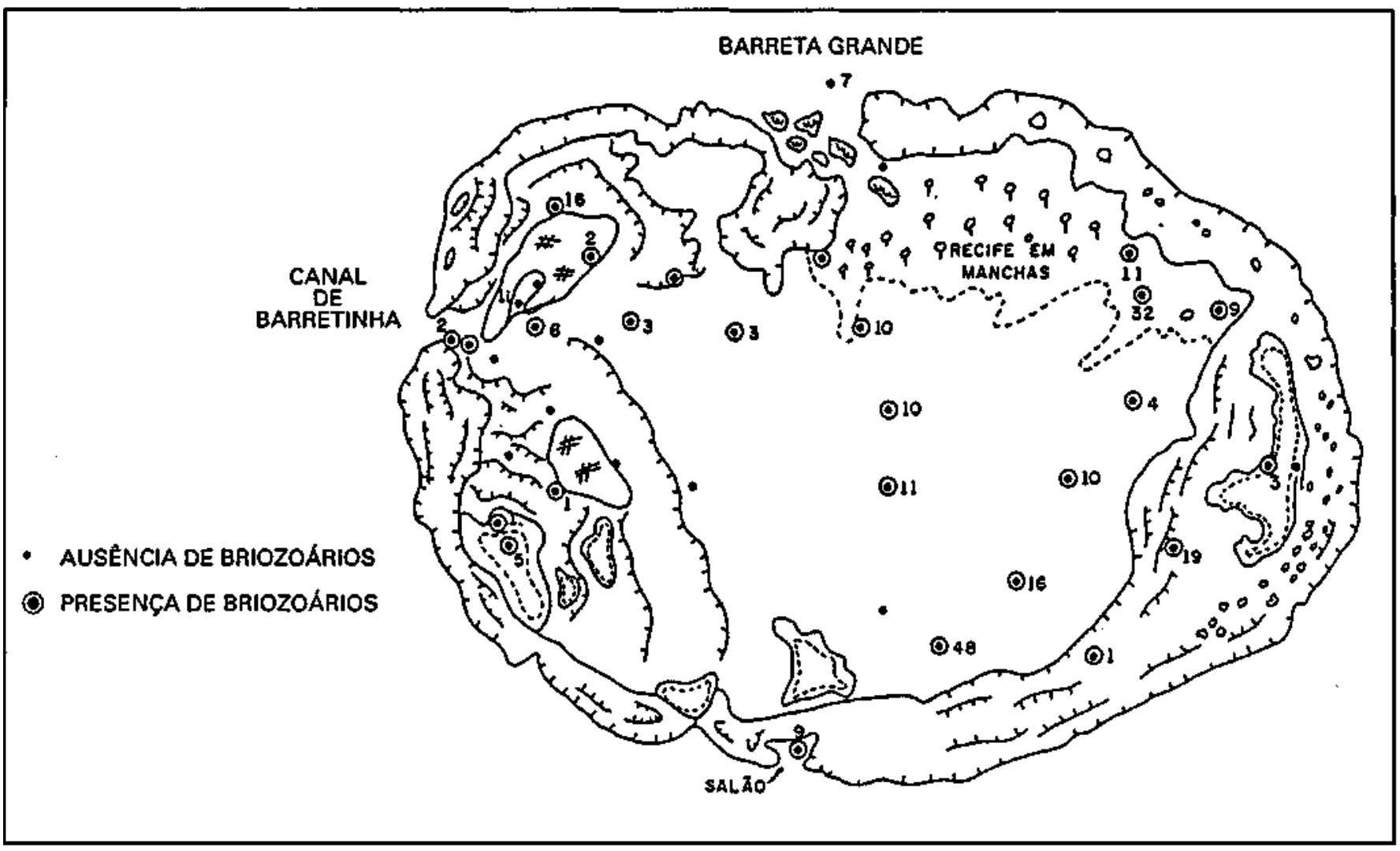

Figura 9 - Principais espécies de foraminiferos do Atol das Rocas Figure 9 - Major species of foraminifera in the Atol das Rocas

das espécies (Tabela 15) indica a predominância de queilostomos dos gêneros Scrupocellaria, Schizoporella, Smittina e Celleporaria, que representam 13\% dos fragmentos analisados (Figura 10). As espécies Onychocella americana e Reptadeonella violácea são também consideradas entre as principais espécies com $5 \%$ de freqüência relativa.

Agrupou-se, sob a denominação de outros, as espécies cujas frequências absoluta e relativa foram inferiores a $2 \%$. Sob esta denominação foram incluídas as espécies: Antropora typica, Hippaliosina imperfecta, Conopeum sp., Metrarabdotos sp. e Escharina pesanseris.

A ordem Cyclostomata, cujos representantes são essencialmente marinhos, está presente em 15 amostras, com uma freqüência relativa importante $(28 \%)$. Dois gêneros foram identificados: Crisia e Nevianipora.

As espécies de briozoários são adaptadas ao ambiente marinho litorâneo e às areias carbonáticas nas proximidades de recifes ou depósitos conchíferos. Elas são características de águas tropicais agitadas (Souza, 1986, 1987 e 1990).

CONCLUSÕES A identificação das espécies de foraminíferos e briozoários nas areias carbonáticas algais do Atol das Rocas mostrou que a área é favorável a estes organismos, devido ao elevado número de espécies isolados de cada grupo.

A microfauna de foraminíferos (68 espécies bentônicas e 05 planctônicas) possui um índice de diversidade elevado $(69 \%)$, muito embora a fauna apresente apenas três espécies dominantes: Archaias angulatus, Amphistegina lessonii e Sorites marginalis. Elas são indicadoras de ambiente marinho normal a hipersalino $(34$ a $37 \%$ c), águas quentes tropicais (variações entre $25^{\circ}$ e $26^{\circ} \mathrm{C}$ ), com substrato de areia carbonática algal.

A dominância das assembléias de Miliolina e Rotaliina confirma que as águas do Atol apresentam hipersalinidade
Tabela 15 - Freqüência relativa das principais espécies de briozoários nas areias carbonáticas do Atol das Rocas Table 15 - Relative abundance of the major bryozoan species in the carbonate sands of the Atol das Rocas

\begin{tabular}{|l|c|c|}
\hline \multicolumn{1}{|c|}{ Especies } & $\begin{array}{c}\text { Frequencia } \\
\text { Absoluta }\end{array}$ & $\begin{array}{c}\text { Frequencia } \\
\text { Relativa (\%) }\end{array}$ \\
\hline Scrupocellaria ssp & 31 & 13 \\
Schizoporella ssp & 22 & 9 \\
Smittina & 15 & 6 \\
Celleporario ssp & 11 & 5 \\
Onychocella americana & 11 & 5 \\
Repladeonella violdced & 11 & 5 \\
Nellia aculata & 08 & 3 \\
Thalamoporella sp & 07 & 3 \\
Schizoporella unicontis & 06 & 3 \\
Cleidochasma porcelana & 04 & 2 \\
Tricellaria sp & 03 & 1 \\
Parasmittina signata & 03 & 1 \\
Rhynchozoon rostrafum $+s p$ & 02 & 1 \\
Steginoporella sp & 02 & 1 \\
Marganetla sp & 02 & 1 \\
Cribrilaria radiata & 02 & 1 \\
Oultos & 05 & 2 \\
Nio identificados & 31 & 13 \\
Sub-lotal - Cheilostomata & 176 & 72 \\
Sub-lotal - Cyclostomata & 69 & 28 \\
\hline Total & 245 & 100 \\
\hline
\end{tabular}

provavelmente periódica, durante as marés baixas e à maior exposição à insolação. Ambiente lagunar raso com águas agitadas é o preferido pela microfauna encontrada. 


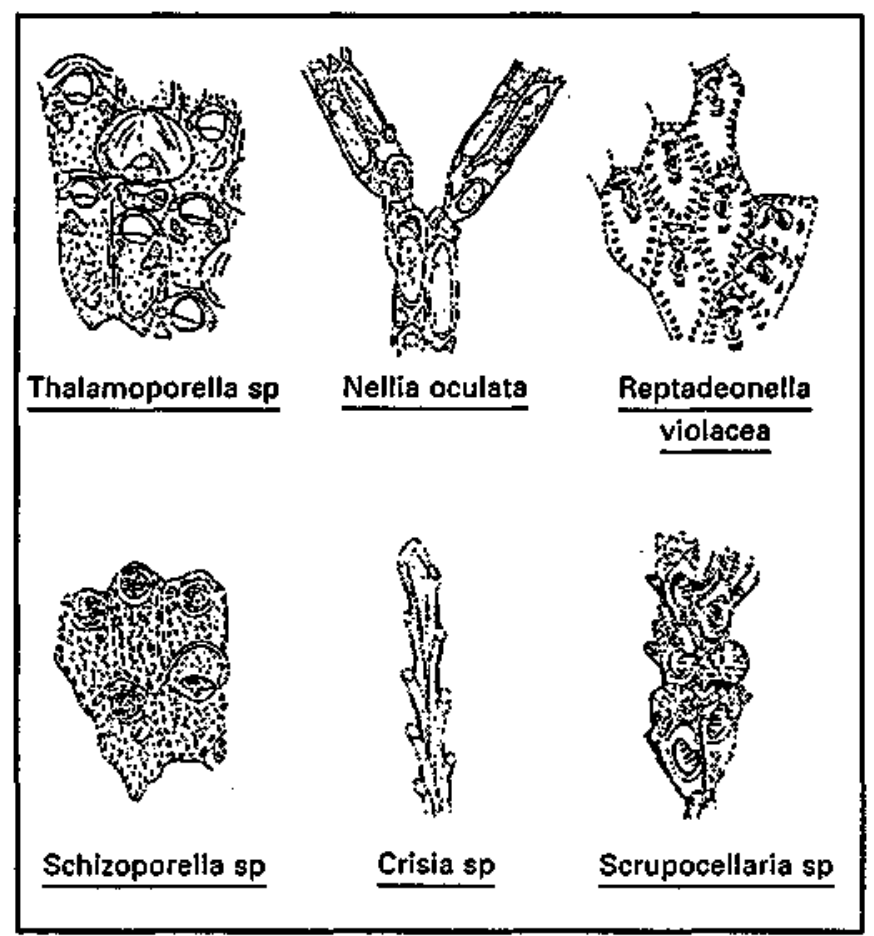

Figura 10 - Principais espécies de briozotrios do Atol das Rocas

Figure 10 - Major species of bryozoans in the Atol das Rocas

Os foraminíferos planctônicos, que não ultrapassam 4\% de freqüência, ocorrem principalmente a NO do Atol, nas proximidades da ilha do Farol e a SO no depósito arenoso. Esta distribuição sugere a presença de águas mais calmas e/ ou mais profundas ocasionais. Globigerinoides trilobus confirma a presença de águas da Corrente do Brasil na área de estudo.

De um modo geral, a população viva, em comparação com o número de exemplares, é muito pequena. Por isso, a relação mortos/vivos, na maioria dos casos, se diferencia muito pouco. A maioria das espécies encontra-se misturada com espécimens mal preservados indicando retrabalhamento e fragmentação dos espécimens. A mistura de formas jovens com formas adultas, mais antigas, evidencia a contemporaneidade do material.
Os estudos da distribuição das espécies de foraminíferos em função da sua localização no Atol mostram que há 5 grupos que apresentam exclusividade para determinado subambiente: a) espécies exclusivas de área de laguna; b) espécies exclusivas de áreas de depósitos arenosos submersos periodicamente; c) espécies exclusivas das piscinas do topo recifal; d) espécies exclusivas do depósito \& laguna, mas ausentes nas piscinas do topo; e) espécies indiferentes às variações do ambiente.

O selecionamento das espécies ocorre também em relação à leste ou oeste do Atol, onde há espécies que ocorrem exclusivamente em um desses lados. Esta distribuição provavelmente está relacionada às variações no ambiente durante o fluxo e refluxo das marés (profundidade, período da submersão, hidrodinâmica, substrato).

A participação dos briozoários como componentes do sedimento é pequena. Apesar do número encontrado de espécies (23) ser relativamente pequeno em comparação com o das espécies de foraminíferos, ele é, ainda, um indicativo de que as condições do ambiente são propícias à fixação da larva e ao desenvolvimento das colônias. Nos recifes costeiros do Banco de Abrolhos, por exemplo, a situação mostra similaridades. A freqüência média dos fragmentos é baixa nos sedimentos que recobrem as áreas recifais com profundidade da água inferior a $5 \mathrm{~m}(<50$ fragmentos $/ 1,00 \mathrm{gr}$ de sedimento), onde as condições de energia do ambiente são altas. Em profundidades maiores, com energia das ondas mais fraca, o número de fragmentos desses organismos é superior (>100 fragmentos/1 OOgr de sedimento) (Souza, trabalho em preparação).

A agitação das águas é o mais provável fator responsável pelo alto grau de fragmentação das colônias, geralmente representadas por 1 ou 2 indivíduos e, no máximo, 5. A predominância de espécies com colônias incrustantes é mais um argumento que confirma a presença desse tipo de hidrodinâmica.

Das 21 espécies isoladas apenas 6 possuem freqüência relativa superior a 5\%: Scrupocellaria spp., Schizoporella ssp., Smittina sp., Celleporaria ssp., Onychocella america$n a$ e Reptadeonella violacea. As espécies são todas típicas de areias carbonáticas e águas rasas. Steginoporella $s p$. e Margaretta $s p$. são indicadoras da presença de suporte algal perecível.

Agradecimentos As autoras agradecem a Ruy Kenji Papa de Kikuchi que gentilmente cedeu as amostras de sedimento para a realização desse trabalho.

\section{REFERÊNCIAS BIBLIOGRÁFICAS}

BOLTOVSKOY, E. 1965 - Los Foraminiferos recientes. Buenos Aires, Eudeba, 510 p., Ill fig.

CUFFEY, R.S. 1972 - The roles of Bryozoans in Modern Coral Reefs Geol. Run. Aschau, v. 61(2): 542-550, Stuttgart.

KIKUCHI, R.K.P \& LEÃO, Z.M.A.N, 1990 - Atol das Rocas: morfologia e características sedimentares. In: CONGR. BRAS. GEOL., Natal. Boi. Resumos, XXXVI Congr. Bras. Geol., SBG, p.45-46.

KIKUCHI, R.K.P. \& ABDON, M.N. 1991 - Mapeamento do Atol das Rocas (reserva biológica - IBAMA/RN), com a utilização de produtos orbitais/Landsat. In: SIMPÓSIO DE GEOLOGIA DO NORDESTE, 14, Recife. Resumos do XIV Simpósio de Geologia do Nordeste, SBG, p. 115-118.

MACHADO, A.J.; SOUZA, F.B.C. \& KIKUCHI, R.K.P. 1991 - Notas sobre organismos incrustantes do Atol das Rocas. In: CONGR. BRÁS. PALEONTOLOGIA, 12, São Paulo. Resumos das Comunicações do XII Congr. Brás. Paleontologia, São Paulo, SP, 1:107.

MACHADO, A.J.; SOUZA, F.B.C. \& KIKUCHI, R.K.P. 1992 - Notas sobre organismos incrustantes do Atol das Rocas. In: CONGR. BRÁS. PALEONTOLOGIA, 12, São Paulo. Anais do XII Congr. Brás. Paleontologia, SBP (no prelo).

MURRAY, J.W. 1973 - Distribution and ecology of living benthic foraminiferida. Crane, Russak and CO, NY, 224 p., 103 fig 12 pi.
SOUZA, F.B.C. 1986 - Distribution et ecologie dês Briozoaires cheilostomes dans lês sediments dês plateformes continentales de Colombie et du Brésil. These de Doutorai en Oceanographie, Univ. Bordeaux I, $353 \mathrm{p}$.

SOUZA, F.B.C. 1987 - Significado ecológico de algumas espécies de briozoários da costa da Bahia. In: SEMINÁRIO UNIVERSITÁRIO DE PESQUISA DE DOCENTES, Salvador, 2. Resumos do XII Seminário Estudantil de Pesquisa, UFBA, p. 309-310.

SOUZA, F.B.C. 1990 - Hidrodinâmica e sedimentação a partir da distribuição dos Briozoários (Área Salvador - Açu da Torre). In: CONG. BRAS. GEOL., 36, Natal, RN Resumos do XXXVI Congr. Brás. Geol., SBG, Natal, RN.

TINOCO, LM. 1966 - Foraminíferos do Atol das Rocas. Trab. Inst. Oceanogr.., Univ. Fed. Pernambuco, Recife (PE). 718:91-114.

TINOCO, LM. 1972 - Foraminíferos dos Bancos da Costa Nordestina, Atol das Rocas e Arquipélago Fernando de Noronha. Trab. Inst. Oceanogr., Univ. Fed. Pernambuco, Recife/PE, 13:49-60 Revisão aceita em 23 de agosto de 1996 